\title{
Implications of the Early X-Ray Afterglow Light Curves of Swift GRBs
}

\author{
Jonathan Granot ${ }^{1}$, Arieh Königl ${ }^{2}$, Tsvi Piran ${ }^{3}$
}

\begin{abstract}
According to current models, gamma-ray bursts (GRBs) are produced when the energy carried by a relativistic outflow is dissipated and converted into radiation. The efficiency of this process, $\epsilon_{\gamma}$, is one of the critical factors in any GRB model. The X-ray afterglow light curves of Swift GRBs show an early stage of flattish decay. This has been interpreted as reflecting energy injection. When combined with previous estimates, which have concluded that the kinetic energy of the late ( $\gtrsim 10 \mathrm{hr}$ ) afterglow is comparable to the energy emitted in $\gamma$-rays, this interpretation implies very high values of $\epsilon_{\gamma}$, corresponding to $\gtrsim 90 \%$ of the initial energy being converted into $\gamma$-rays. Such a high efficiency is hard to reconcile with most models, including in particular the popular internal-shocks model. We re-analyze the derivation of the kinetic energy from the afterglow X-ray flux and re-examine the resulting estimates of the efficiency. We confirm that, if the flattish decay arises from energy injection and the pre-Swift broad-band estimates of the kinetic energy are correct, then $\epsilon_{\gamma} \gtrsim 0.9$. We discuss various issues related to this result, including an alternative interpretation of the light curve in terms of a two-component outflow model, which we apply to the X-ray observations of GRB 050315. We point out, however, that another interpretation of the flattish decay - a variable X-ray afterglow efficiency (e.g., due to a time dependence of afterglow shock microphysical parameters) — is possible. We also show that direct estimates of the kinetic energy from the late X-ray afterglow flux are sensitive to the assumed values of the shock microphysical parameters and suggest that broad-band afterglow fits might have underestimated the kinetic energy (e.g., by overestimating the fraction of electrons that are accelerated to relativistic energies). Either one of these possibilities implies a lower $\gamma$-ray efficiency, and their joint effect could conceivably reduce the estimate of the typical $\epsilon_{\gamma}$ to a value in the range $\sim 0.1-0.5$.
\end{abstract}

\footnotetext{
${ }^{1}$ KIPAC, Stanford University, P.O. Box 20450, MS 29, Stanford, CA 94309; granot@slac.stanford.edu

${ }^{2}$ Department of Astronomy and Astrophysics and Enrico Fermi Institute, University of Chicago, 5640 South Ellis Avenue, Chicago, IL 60637; arieh@jets.uchicago.edu

${ }^{3}$ Racah Institute of Physics, The Hebrew University, Jerusalem, 91904, Israel; tsvi@phys.huji.ac.il
} 
Subject headings: gamma-rays: bursts — ISM: jets and outflows — radiation mechanisms: nonthermal — X-rays: individual (GRB 050315)

\section{Introduction}

Recent observations by the Swift X-ray telescope have provided new information on the early behavior of the X-ray light curve of long-duration ( $\gtrsim 2$ s) gamma-ray burst (GRB) sources. Specifically, it was found (Nousek et al. 2005) that the light curves of these sources have a generic shape consisting of three distinct power-law segments $\propto t^{-\alpha}$ : an initial (at $t<t_{\text {break }, 1}$, with $300 \mathrm{~s} \lesssim t_{\text {break,1 }} \lesssim 500 \mathrm{~s}$ ) very steep decline with time $t$ (with a power-law index $\alpha_{1}$ in the range $3 \lesssim \alpha_{1} \lesssim 5$; see also Tagliaferri et al. 2005 and Bartherlmy et al. 2005 ); a subsequent (at $t_{\text {break }, 1}<t<t_{\text {break }, 2}$, with $10^{3} \mathrm{~s} \lesssim t_{\text {break }, 2} \lesssim 10^{4} \mathrm{~s}$ ) very shallow decay $\left(0.2 \lesssim \alpha_{2} \lesssim 0.8\right)$; and a final steepening (at $\left.t>t_{\text {break }, 2}\right)$ to the canonical power-law behavior $\left(1 \lesssim \alpha_{3} \lesssim 1.5\right)$ that was known from pre-Swift observations.

Nousek et al. (2005) already recognized that these results have direct consequences to the question of the $\gamma$-ray emission efficiency in GRB sources. This question is important to our understanding of the basic prompt-emission mechanism. In the currently accepted interpretation (e.g., Piran 1999, 2004), the $\gamma$-rays originate in a relativistic jet that is launched from the vicinity of a newly born neutron star or stellar-mass black hole. In the simplest picture, a fraction $\epsilon_{\gamma}$ of the energy injected at the source is given to the prompt radiation, with the remaining fraction $\left(1-\epsilon_{\gamma}\right)$ ending up as kinetic energy of ambient gas that is swept up by a forward shock and then mostly radiated as early afterglow emission. One attractive mechanism for explaining the prompt emission characteristics invokes internal shocks that are generated when the ejecta have a nonuniform distribution of Lorentz factors, which results in outflowing "shells" colliding with each other at large distances from the source.

Pre-Swift observations, based on measurements of the $\gamma$-ray fluence and of the "late" ( $\gtrsim 10 \mathrm{hr}$ ) afterglow emission, have implied (when interpreted in the context of the basic jet model) comparable (and narrowly clustered) values for the radiated $\gamma$-ray energy and the kinetic energy feeding the afterglow emission, i.e., $\epsilon_{\gamma} \sim 0.5$ (e.g., Frail et al. 2001; Panaitescu \& Kumar 2001a,b, 2002; Berger et al. 2003; Bloom et al. 2003; Yost et al. 2003). This result is seemingly problematic for the internal-shocks model, for which an order-ofmagnitude lower value for $\epsilon_{\gamma}$ is a more natural expectation (Kobayashi et al. 1997; Daigne \& Mochkovitch 1998; Kumar 1999; Guetta et al. 2001). This apparent difficulty could in principle be overcome if the ejected shells have a highly nonuniform distribution of Lorentz factors (e.g., Beloborodov 2000; Kobayashi \& Sari 2001). However, to fit the data the shells must also satisfy a number of other restrictive conditions, which reduces the attractiveness 
of this interpretation (see $\S 5$ ). An alternative proposal was made by Peng et al. (2005), who argued that if the jet consists of an ultra-relativistic narrow component (from which the prompt emission originates) and a moderately relativistic wide component, with the latter having a higher kinetic energy and the former a higher kinetic energy per unit solid angle, then the wide component would dominate the late afterglow emission and the $\gamma$-ray radiative efficiency of the narrow component could be significantly lower than the value of $\epsilon_{\gamma}$ inferred under the assumption of a single-component jet. As explained in Peng et al. (2005), this proposal was motivated by observational indications of the presence of two components in the late afterglow light curves of several GRB sources and by the predictions of certain GRB source models.

The earliest (steepest) segment of the afterglow light curve is most naturally explained as radiation at large angles to our line of sight corresponding either to the prompt emission (Kumar \& Panaitescu 2000) or to emission from the reverse shock that is driven into the ejecta (Kobayashi et al. 2005). This implies that the early afterglow emission is much weaker than what would be expected on the basis of an extrapolation from the late-afterglow data. This behavior was interpreted by Nousek et al. (2005) as an indication of an even higher $\gamma$-ray emission efficiency, typically $\epsilon_{\gamma} \sim 0.9$. Such a high efficiency could render the internal-shocks model untenable.

Our primary goal is to evaluate the prompt-emission efficiency as accurately and systematically as possible on the basis of current data. For this purpose we re-derive in $\S 2.1$ expressions that explicitly relate $\epsilon_{\gamma}$ to observable quantities. In particular, we express $\epsilon_{\gamma}$ in terms of the product $\kappa f$ of two parameters, one $(\kappa)$ encapsulating information that could be obtained by pre-Swift observations, and the other $(f)$ representing early-time data obtained in Swift measurements. In $\S 2.2$ we re-examine the estimates of the kinetic energy during the afterglow phase as inferred from the X-ray flux and present a new general formulation, correcting errors that have propagated in the literature and have generally led to an underestimate of the kinetic energy. We then analyze both pre-Swift ( $\S 2.3$ ) and Swift ( $\S 2.4$ ) data in a uniform manner in the context of this formalism. We argue that the kinetic energy estimates remain subject to considerable uncertainties. In particular, while the simple analysis of a large number of bursts (using "typical" values for the microphysical parameters) yields rather high values for the kinetic energy and hence a low inferred $\gamma$-ray efficiency, a multiwavelength analysis (which determines the microphysical parameters from the fit to the data) of a small subset suggests that the kinetic energy is lower and hence the inferred value of $\epsilon_{\gamma}$ is higher. Some caveats to this analysis are considered in $\S 2.5$.

The suggestion that the Swift observations imply a higher value of $\epsilon_{\gamma}$ in comparison with the pre-Swift results is based on the interpretation of the flattish segment of the X-ray light 
curve as reflecting an increase in the kinetic energy of the forward shock during the early stages of the afterglow. In this picture, the kinetic energy just after the prompt-emission phase was significantly lower than the kinetic energy estimated from the later stages of the afterglow (the pre-Swift results). One conceivable way of avoiding the need to increase the estimate of $\epsilon_{\gamma}$ in light of the new Swift observations is through a time evolution (specifically, an increase with $t$ ) of the X-ray afterglow emission efficiency $\epsilon_{\mathrm{X}}$. Such a behavior could in principle also account for the flattish segment of the light curve and eliminate the need to invoke an increase in the shock kinetic energy. If, in addition, the value of the afterglow kinetic energy at late times were underestimated by the broad-band fits to pre-Swift GRB afterglows, which could be the case if only a fraction $\xi_{e}<1$ of the electrons were accelerated to relativistic energies in the afterglow shock (see Eichler \& Waxman 2005), then the typical afterglow efficiency would be further reduced (to a value as low as $\epsilon_{\gamma} \sim 0.1$ if $\xi_{e} \sim 0.1$ ), which might reconcile the new Swift data with the comparatively low efficiencies expected in the internal-shocks model. We discuss these issues in $\S 3$.

A two-component jet model with the characteristics required for reducing the inferred $\gamma$-ray emission efficiency is evidently disfavored by the Swift data, but a model of this type with different parameters could provide an alternative interpretation of the flattish shape of the light curve between $t_{\mathrm{break}, 1}$ and $t_{\mathrm{break}, 2}$. We elaborate on these matters in $\S 4$, where we also present a tentative fit to the X-ray light curve of the Swift source GRB 050315 in the context of this scenario.

Our conclusions on the physical implications of the early X-ray light-curve observations of GRB sources are presented in $\S 5$.

\section{Estimating the Gamma-Ray Efficiency}

The observed isotropic-equivalent luminosity can generally be expressed as $L_{\text {iso }}=\epsilon E_{\text {iso }} / T$, where $E_{\text {iso }}$ is the isotropic-equivalent energy of the relevant component, $T$ is the duration of the relevant emission, and $\epsilon$ is the overall efficiency. This efficiency is a product of several factors: $\epsilon=\epsilon_{\mathrm{dis}} \epsilon_{e} \epsilon_{\mathrm{rad}} \epsilon_{\mathrm{obs}}$, where a fraction $\epsilon_{\mathrm{dis}}$ of the total energy is dissipated into internal energy, a fraction $\epsilon_{e}$ of the internal energy goes into electrons (or positrons) and can in principle be radiated away, a fraction $\epsilon_{\text {rad }}$ of the electron energy is actually radiated, and a fraction $\epsilon_{\mathrm{obs}}$ of the radiated energy falls within the observed range of photon energies. A fraction $\epsilon_{\gamma}=\epsilon_{\mathrm{dis}, \mathrm{GRB}} \epsilon_{e, \mathrm{GRB}} \epsilon_{\mathrm{rad}, \mathrm{GRB}}$ of the total original (isotropic equivalent) energy $E_{\mathrm{iso}, 0}$ is radiated away during the prompt emission, $E_{\gamma, \text { iso }}=\epsilon_{\gamma} E_{\text {iso }, 0}$, while the remaining (kinetic) energy in the $\gamma$-ray emitting component of the outflow, $E_{\mathrm{k}, \text { iso }, 0}=\left(1-\epsilon_{\gamma}\right) E_{\mathrm{iso}, 0}$, is transferred to the afterglow shock at $t_{\mathrm{dec}}$. In the prompt GRB emission $\epsilon_{\mathrm{GRB}}=\epsilon_{\gamma} \epsilon_{\mathrm{obs}, \mathrm{GRB}}$. In the 
afterglow $\epsilon_{\mathrm{dis}, \mathrm{X}} \approx 1$, and therefore $\epsilon_{\mathrm{X}} \approx \epsilon_{e, \mathrm{X}} \epsilon_{\mathrm{rad}, \mathrm{X}} \epsilon_{\mathrm{obs}, \mathrm{X}}$, where we concentrate on the X-ray afterglow.

Using the above expression for $\epsilon_{\gamma}$, it becomes clearer why it is difficult for it to assume very high $(\gtrsim 0.9)$ values. Whereas $\epsilon_{\mathrm{rad}, \mathrm{GRB}} \approx 1$ is possible, and even expected if the electrons cool significantly over a dynamical time (as is typically expected in the internal-shocks model $), \epsilon_{\gamma} \gtrsim 0.9$ requires in addition that both $\epsilon_{\mathrm{dis}, \mathrm{GRB}}>0.9$ and $\epsilon_{e, \mathrm{GRB}}>0.9$. It is difficult to achieve $\epsilon_{\text {dis, GRB }}>0.9$ (i.e., dissipate more than $90 \%$ of the total energy) in most models for the prompt emission, and in particular in the internal-shocks model. Furthermore, it is not trivial to produce $\epsilon_{e, \mathrm{GRB}}>0.9$ (i.e. more than $90 \%$ of the postshock energy going to electrons) in the internal-shocks model, where the electrons are believed to be accelerated in a shock propagating into a magnetized proton-electron plasma. In particular, this would require that less than $10 \%$ of the postshock energy goes into the protons and the magnetic field, which is difficult since the protons carry most of the energy (in kinetic form) and the magnetic field likely carries a comparable energy in the upstream fluid ahead of the shock. It might conceivably be possible if less than $10 \%$ of the energy is in the magnetic field and the protons can somehow transfer their energy to the electrons, which radiate it away.

\subsection{Relationship to Observed Quantities}

The X-ray afterglow isotropic-equivalent luminosity, $L_{\mathrm{X} \text {,iso }}$, can be expressed in terms of the X-ray afterglow flux, $F_{\mathrm{X}}$,

$$
L_{\mathrm{X}, \text { iso }}(t)=4 \pi d_{L}^{2}(1+z)^{\beta-\alpha-1} F_{\mathrm{X}}(t)
$$

if $F_{\nu} \propto \nu^{-\beta} t^{-\alpha}$ in the relevant ranges in frequency and time. ${ }^{1}$ The efficiency of the X-ray afterglow emission is defined as

$$
\epsilon_{\mathrm{X}}(t) \equiv \frac{t L_{\mathrm{X}, \mathrm{iso}}(t)}{E_{\mathrm{k}, \text { iso }}(t)}
$$

where $E_{\mathrm{k}, \text { iso }}$ is the isotropic-equivalent kinetic energy in the afterglow shock.

Using the relation $E_{\mathrm{iso}, 0}=E_{\gamma, \text { iso }} / \epsilon_{\gamma}=E_{\mathrm{k}, \text { iso }, 0} /\left(1-\epsilon_{\gamma}\right)$, we obtain

$$
\frac{\epsilon_{\gamma} \epsilon_{\mathrm{obs}, \mathrm{GRB}}}{\left(1-\epsilon_{\gamma}\right)}=\frac{E_{\gamma, \text { iso }}^{\mathrm{obs}}}{E_{\mathrm{k}, \mathrm{iso}, 0}}=\kappa f \quad, \quad \kappa \equiv \frac{E_{\gamma, \text { iso }}^{\mathrm{obs}}}{E_{\mathrm{k}, \text { iso }}\left(t_{*}\right)} \quad, \quad f \equiv \frac{E_{\mathrm{k}, \mathrm{iso}}\left(t_{*}\right)}{E_{\mathrm{k}, \mathrm{iso}, 0}}
$$

\footnotetext{
${ }^{1}$ For the more general case, see eq. [1] of Nousek et al. (2005) and the discussion thereafter. Here $L_{\mathrm{X} \text {,iso }}(t)=\int_{\nu_{1}}^{\nu_{2}} d \nu L_{\nu \text {,iso }}(t)$, where both $\nu$ and $t$ are measured in the cosmological frame of the GRB, whereas $F_{\mathrm{X}}(t)=\int_{\nu_{1}}^{\nu_{2}} d \nu F_{\nu}(t)$, where both $\nu$ and $t$ are measured in the observer frame.
} 
where $t_{*}$ can be chosen as a time at which it is convenient to estimate $E_{\mathrm{k}, \text { iso }}$, and we shall use $t_{*}=10 \mathrm{hr}$ (since it is widely used in the literature and is typically $>t_{\mathrm{break}, 2}$, the end of the flattish segment of the X-ray light curve).

An important question is how to estimate $f$ and $\kappa$ from observations. The most straightforward way of estimating $f=E_{\mathrm{k}, \text { iso }}(10 \mathrm{hr}) / E_{\mathrm{k}, \text { iso }, 0}$, which has been used by Nousek et al. (2005), is by evaluating the value of the X-ray flux decrement at $t_{\text {dec }}$ relative to the extrapolation to $t_{\mathrm{dec}}$ of the late-time $\left(t>t_{\mathrm{break}, 2}\right)$ flux, and translating this flux ratio into an energy ratio according to the standard afterglow theory. When estimating $f$ in this way we assume that

$$
\epsilon_{\mathrm{X}} \sim(1+Y)^{-1} \epsilon_{e}\left(\nu_{m} / \nu_{\mathrm{X}}\right)^{(p-2) / 2}
$$

where $\nu_{m}$ and $\nu_{c}$ are the characteristic synchrotron frequency and cooling break frequency, respectively (Sari et al. 1998; Granot \& Sari 2002) and $Y$ is the Compton y-parameter. This result can also be obtained under the assumptions of standard afterglow theory, as follows. The overall X-ray afterglow efficiency can be written as $\epsilon_{\mathrm{X}} \approx \epsilon_{e} \epsilon_{\mathrm{rad}} \epsilon_{\mathrm{obs}}$, where $\epsilon_{\mathrm{rad}} \approx \min \left[1,\left(\nu_{m} / \nu_{c}\right)^{(p-2) / 2}\right]$ and $\epsilon_{\mathrm{obs}} \approx(1+Y)^{-1} \max \left[\left(\nu_{m} / \nu_{\mathrm{X}}\right)^{(p-2) / 2},\left(\nu_{c} / \nu_{\mathrm{X}}\right)^{(p-2) / 2}\right]$; the factor of $(1+Y)^{-1}$ is the fraction of the radiated energy in the synchrotron component, and it is present because the synchrotron self-Compton (SSC) component typically does not contribute significantly to $F_{\mathrm{X}}(10 \mathrm{hr})$ but may still dominate the total radiated luminosity. The factor $(1+Y)^{-1}$ is generally assumed to be small, consistent with the usual inference that the magnetic-to-thermal energy ratio in the emission region, $\epsilon_{B}$, is smaller than $\epsilon_{e}$ (see eq. [12]). ${ }^{2}$ However, one should keep in mind that a different time dependence of $\epsilon_{\mathrm{X}}$ (which might occur under less standard assumptions) would modify the value of $f$ accordingly. The value of $\epsilon_{\mathrm{obs}, \mathrm{GRB}}$ can be estimated by extrapolating the observed part of the spectrum and modeling additional spectral components that might carry considerable energy.

To estimate $\kappa=E_{\gamma, \text { iso }}^{\text {obs }} / E_{\mathrm{k}, \text { iso }}(10 \mathrm{hr})$ we calculate $E_{\gamma, \text { iso }}^{\text {obs }}=f_{\gamma} 4 \pi d_{L}^{2}(1+z)^{-1}$ directly from the observed $\gamma$-ray fluence, $f_{\gamma}$, and the measured redshift, $z$. The denominator, $E_{\mathrm{k} \text {,iso }}(10 \mathrm{hr})$, has been estimated following Freedman \& Waxman (2001) and Kumar (2000) (see also Lloyd-Ronning \& Zhang 2004) from $F_{\mathrm{X}}(10 \mathrm{hr})$ using standard afterglow theory. We reconsider this calculation in the next section.

\footnotetext{
${ }^{2}$ At early times (less than about a day) inverse-Compton radiation is important in cooling the electrons that are emitting synchrotron X-rays. Later the X-ray emitting electrons are within the Klein-Nishina cutoff and are not cooled by inverse-Compton radiation (see Fan \& Piran 2006).
} 


\subsection{Estimates of the kinetic energy from the X-ray afterglow observations}

Using the results of Granot \& Sari (2002) we find that, if $\nu_{\mathrm{X}}>\max \left(\nu_{m}, \nu_{c}\right)$, we can estimate the kinetic energy from the observed X-ray flux. We use

$$
E_{\mathrm{k}, \text { iso }}(t)=9.2 \times 10^{52} \frac{g(p)}{g(2.2)}(1+Y)^{4 /(p+2)} \epsilon_{e,-1}^{4(1-p) /(p+2)} \epsilon_{B,-2}^{(2-p) /(p+2)} L_{\mathrm{X}, 46}^{4 /(p+2)} t_{10 \mathrm{hr}}^{(3 p-2) /(p+2)} \mathrm{erg},
$$

where $L_{\mathrm{X} \text {,iso }}=L_{\mathrm{X}, 46} 10^{46} \mathrm{erg} \mathrm{s}^{-1}$ is the isotropic-equivalent $\mathrm{X}$-ray luminosity in the range $2-10 \mathrm{keV}$ at a time $10 t_{10 \mathrm{hr}} \mathrm{hr}$, both measured in the cosmological frame of the GRB [corresponding to an observed time of $t=10(1+z) t_{10 \mathrm{hr}} \mathrm{hr}$ and spectral range $2 /(1+z)-$ $10 /(1+z) \mathrm{keV}], \epsilon_{e,-1}=\epsilon_{e} / 0.1, \epsilon_{B,-2}=\epsilon_{B} / 0.01$, and

$$
g(p)=\left[\frac{(p-1)^{p-1} \exp (5.89 p-12.7)}{\left(5^{(p-2) / 2}-1\right)(p-2)^{p-2}(p-0.98)}\right]^{4 /(p+2)} .
$$

In equation (5), $L_{\mathrm{X} \text {,iso }}$ is evaluated through $F_{\mathrm{X}}$ using equation (1), and it is assumed that $F_{\mathrm{X}}$ is dominated by the synchrotron component. If there is a significant contribution to $F_{\mathrm{X}}\left(t_{*}\right)$ from the SSC component then equation (5) would overestimate $E_{\mathrm{k} \text {,iso }}\left(t_{*}\right)$, but the correct estimate could still be obtained if only the synchrotron contribution to $F_{\mathrm{X}}\left(t_{*}\right)$ is used (although in practice it might be difficult and somewhat model-dependent to disentangle the synchrotron and SSC components). For our fiducial parameters $(1+Y) \approx 3.7$, so the numerical coefficient in equation (5) is $3.2 \times 10^{53} \mathrm{erg}$ for $p=2.2$ and $5.8 \times 10^{53} \mathrm{erg}$ for $p=2.5$. This is a factor $\sim 30-60$ higher than the numerical coefficient in equation (7) of Lloyd-Ronning \& Zhang (2004). Part of this difference (a factor of $\sim 3-3.5$ ) reflects the fact that these authors did not take into account the SSC contribution, which reduces the flux in the relevant power-law segment of the spectrum by a factor of $(1+Y)$ (Sari \& Esin 2001; Granot \& Sari 2002), ${ }^{3}$ but this does not account for most of the discrepancy. Most of the difference is basically a result of a higher (by a factor of $\sim 38$ for $p=2.2$ ) value of $\nu_{m}$ that Lloyd-Ronning \& Zhang (2004) use in their equation (2), given that their expressions for $\nu_{c}$ and $F_{\nu, \max }$ (their eqs. [3] and [4]) are very similar to those in Granot \& Sari (2002) and that $F_{\nu>\max \left(\nu_{m}, \nu_{c}\right)}=F_{\nu, \max } \nu_{c}^{1 / 2} \nu_{m}^{(p-1) / 2} \nu^{-p / 2} \propto \nu_{m}^{(p-1) / 2}$. Our numerical coefficient is lower than that in equation (4) of Freedman \& Waxman (2001) by a factor of $\sim 3$ and $\sim 8$ (or $\sim 12$ and $\sim 31$ if SSC is not taken into account) for $p=2.2$ and $p=2.5$, respectively. The difference in the numerical coefficient between Lloyd-Ronning \& Zhang (2004) and Freedman \& Waxman (2001) is by a factor of $\sim 100$ and $\sim 475$ for $p=2.2$ and $p=2.5$, respectively.

\footnotetext{
${ }^{3}$ This is assuming that the SSC component does not contribute considerably to the observed X-ray flux, which is typically the case at $t_{*}=10 \mathrm{hr}$.
} 
Using equations (2) and (5), we can express the efficiency as

$$
\begin{aligned}
\epsilon_{\mathrm{X}}(t) & =3.5 \times 10^{-3}\left[\frac{g(p)}{g(2.2)}\right]^{-(p+2) / 4}(1+Y)^{-1} \epsilon_{e,-1}^{p-1} \epsilon_{B,-2}^{(p-2) / 4} E_{\mathrm{k}, \mathrm{iso}, 52}^{(p-2) / 4} t_{10 \mathrm{hr}}^{-3(p-2) / 4} \\
& =3.9 \times 10^{-3} \frac{g(2.2)}{g(p)}(1+Y)^{-4 /(p+2)} \epsilon_{e,-1}^{4(p-1) /(p+2)} \epsilon_{B,-2}^{(p-2) /(p+2)} L_{\mathrm{X}, 46}^{(p-2) /(p+2)} t_{10 \mathrm{hr}}^{-2(p-2) /(p+2)}
\end{aligned}
$$

where $E_{\mathrm{k}, \text { iso }, 52}=E_{\mathrm{k}, \text { iso }}(t) /\left(10^{52} \mathrm{erg}\right)$. For our fiducial values (and for $L_{\mathrm{X}, 46}$ rather than $\left.E_{\mathrm{k}, \text { iso }, 52}\right)$, the numerical coefficient in equation $(7)$ is $1.1 \times 10^{-3}$ for $p=2.2$ and $6.3 \times 10^{-4}$ for $p=2.5$.

Equations (5)-(7) are valid for $p>2$, but they can be easily generalized to $p \lesssim 2$ by substituting $\epsilon_{e} \rightarrow \bar{\epsilon}_{e}(p-1) /(p-2)$, where $\bar{\epsilon}_{e}=\epsilon_{e} \gamma_{\min } /\left\langle\gamma_{e}\right\rangle,\left\langle\gamma_{e}\right\rangle=\int d \gamma_{e}\left(d N / d \gamma_{e}\right) \gamma_{e}\left[\int d \gamma_{e}\left(d N / d \gamma_{e}\right)\right]^{-1}$ is the average electron Lorentz factor, and the electron energy distribution is a power law of index $p$ at low energies above $\gamma_{\text {min }}$. If the electron energy distribution is a single power law, $d N / d \gamma_{e} \propto \gamma_{e}^{-p}$ for $\gamma_{\min }<\gamma_{e}<\gamma_{\max }$, then

$$
\frac{\epsilon_{e}}{\bar{\epsilon}_{e}}=\frac{\left\langle\gamma_{e}\right\rangle}{\gamma_{\min }}=\left(\frac{p-1}{p-2}\right) \frac{1-\left(\gamma_{\max } / \gamma_{\min }\right)^{2-p}}{1-\left(\gamma_{\max } / \gamma_{\min }\right)^{1-p}}=\left\{\begin{array}{cc}
\approx(p-1) /(p-2) & p>2, \\
\ln \left(\gamma_{\max } / \gamma_{\min }\right) & p=2, \\
\left(\gamma_{\max } / \gamma_{\min }\right)^{2-p}(p-1) /(2-p) & 1<p<2, \\
\approx\left(\gamma_{\max } / \gamma_{\min }\right) / \ln \left(\gamma_{\max } / \gamma_{\min }\right) & p=2, \\
\left(\gamma_{\max } /(2-p)\right. & p<1 .
\end{array}\right.
$$

This results in slightly modified equations:

$$
\begin{gathered}
E_{\mathrm{k}, \text { iso }}(t)=1.19 \times 10^{52} \frac{\bar{g}(p)}{\bar{g}(2.2)}(1+Y)^{4 /(p+2)} \bar{\epsilon}_{e,-1}^{4(1-p) /(p+2)} \epsilon_{B,-2}^{(2-p) /(p+2)} L_{\mathrm{X}, 46}^{4 /(p+2)} t_{10 \mathrm{hr}}^{(3 p-2) /(p+2)} \mathrm{erg}, \\
\bar{g}(p)=\left[\frac{(p-2) \exp (5.89 p-12.7)}{\left(5^{(p-2) / 2}-1\right)(p-0.98)}\right]^{4 /(p+2)}, \\
\epsilon_{\mathrm{X}}(t)=3.01 \times 10^{-2}\left[\frac{\bar{g}(p)}{\bar{g}(2.2)}\right]^{-(p+2) / 4}(1+Y)^{-1} \bar{\epsilon}_{e,-1}^{p-1} \bar{\epsilon}_{B,-2}^{(p-2) / 4} E_{\mathrm{k}, \mathrm{iso}, 52}^{(p-2) / 4} t_{10 \mathrm{hr}}^{-3(p-2) / 4} \\
=3.03 \times 10^{-3} \frac{\bar{g}(2.2)}{\bar{g}(p)}(1+Y)^{-4 /(p+2)} \bar{\epsilon}_{e,-1}^{4(p-1) /(p+2)} \epsilon_{B,-2}^{(p-2) /(p+2)} L_{\mathrm{X}, 46}^{(p-2) /(p+2)} t_{10 \mathrm{hr}}^{-2(p-2) /(p+2)}
\end{gathered}
$$

Note that the numerical coefficient was calculated in Granot \& Sari (2002) only for $p=$ 2.2, 2.5, 3 and interpolated between these values. Extrapolating that formula to $p \lesssim 2$ could potentially be very inaccurate. 
For simplicity we use the expression for $Y$ that is valid in the fast-cooling regime,

$$
Y=\frac{\left(1+4 \epsilon_{e} / \epsilon_{B}\right)^{1 / 2}-1}{2} \approx\left\{\begin{array}{cc}
\left(\epsilon_{e} / \epsilon_{B}\right)^{1 / 2} & \epsilon_{e} / \epsilon_{B} \gg 1 \\
\epsilon_{e} / \epsilon_{B} & \epsilon_{e} / \epsilon_{B} \ll 1
\end{array},\right.
$$

which is still reasonable at $10 \mathrm{hr}$, even if it is slightly after the transition to slow cooling. More generally, $\epsilon_{e} / \epsilon_{B}$ should be multiplied by $\epsilon_{\mathrm{rad}} \approx \min \left[1,\left(\nu_{m} / \nu_{c}\right)^{(p-2) / 2}\right]$, where for $p<2$ and $\nu_{c}<\nu_{\text {max }}$ we have $\epsilon_{\text {rad }} \approx 1$.

Before applying these relations to observed bursts we remark on a common misconception concerning the dependence of $E_{\mathrm{k} \text {,iso }}$ that is inferred from $F_{\mathrm{X}}$ on $\epsilon_{B}$ and $\epsilon_{e}$. It has been argued that $E_{\mathrm{k}, \text { iso }}$ is very insensitive to the exact value of $\epsilon_{B}$ (e.g., Freedman \& Waxman 2001; Piran et al. 2001). This follows from the observation (see eq. [5]) that for $\nu_{\mathrm{X}}>\max \left(\nu_{m}, \nu_{c}\right)$, $E_{\mathrm{k}, \text { iso }} \propto(1+Y)^{4 /(p+2)} \epsilon_{e}^{-4(p-1) /(p+2)} \epsilon_{B}^{-(p-2) /(p+2)}$, which suggests that $E_{\mathrm{k}, \text { iso }}$ depends very weakly on $\epsilon_{B}$. However, this holds only in the limit where $(1+Y) \approx 1$, which corresponds to $Y \ll 1$ and $\epsilon_{B} \gg \epsilon_{e}$, whereas observations suggest that the opposite limit typically applies, $\epsilon_{B} \ll \epsilon_{e}$, in which case $(1+Y) \approx Y \approx\left(\epsilon_{e} / \epsilon_{B}\right)^{1 / 2}$ and $E_{\mathrm{k}, \text { iso }} \propto \epsilon_{B}^{-p /(p+2)}$. This is a significantly stronger dependence on $\epsilon_{B}$. (Note that the inferred value of $\epsilon_{B}$ varies by $\sim 2$ orders of magnitude among different afterglows, from $\sim 10^{-3}$ to $\sim 0.1$, corresponding to a change of more than an order of magnitude in the estimated value of $E_{\mathrm{k}, \text { iso }}$.) The dependence of $E_{\mathrm{k}, \text { iso }}$ on $\epsilon_{e}$ is stronger, $\propto \epsilon_{e}^{-4(p-1) /(p+2)}$ (i.e., slightly steeper than an inverse linear relation) in the limit $\epsilon_{B} \gg \epsilon_{e}$, but only $\propto \epsilon_{e}^{-2(2 p-3) /(p+2)}$ in the more relevant limit of $\epsilon_{B} \ll \epsilon_{e}$. In the latter case the dependence of $E_{\mathrm{k}, \text { iso }}$ on $\epsilon_{B}$ is rather similar to its dependence on $\epsilon_{e}{ }^{4}$ However, $\epsilon_{e}$ appears to vary much less than $\epsilon_{B}$ among different afterglows, only covering a range of about one order of magnitude (between $\sim 10^{-1.5}$ and $\sim 10^{-0.5}$ ), which corresponds to a variation in $E_{\mathrm{k}, \text { iso }}$ by a factor of $\sim 4$. It is also worth noting that the expression for $E_{\mathrm{k}, \text { iso }}$ has some (nontrivial) dependence on the value of $p$ (see eq. [6]).

\subsection{Pre-Swift GRBs}

Table 1 shows the estimated values of $\kappa$ deduced from the observational properties of 17 pre-Swift GRBs with known redshifts, using the samples of Berger et al. (2003) and Bloom et al. (2003). We provide the values $E_{\mathrm{k}, \text { iso }, 10 \mathrm{hr}}=E_{\mathrm{k}, \text { iso }}(10 \mathrm{hr})$ and $\kappa$ for our fiducial parameter values $\left(\epsilon_{e}=0.1, \epsilon_{B}=0.01\right.$, and $\left.p=2.2\right)$. The value of $E_{\mathrm{k}, \text { iso, } 10 \mathrm{hr}}$ and therefore

\footnotetext{
${ }^{4}$ Quite often $Y \sim 1-2$ is inferred, in which case neither of the asymptotic limits $Y \ll 1$ and $Y \gg 1$ is applicable and the dependence of $E_{\mathrm{k} \text {,iso }}$ on $\epsilon_{B}$ and $\epsilon_{e}$ does not have a power-law form but rather the more complex form given by eqs. (5) and (12).
} 
of $\kappa$ depends on the values of the microphysical parameters $\left(\epsilon_{e}, \epsilon_{B}\right.$, and $\left.p\right)$ that are not well known. Therefore we also calculate $E_{\mathrm{k}, \text { iso, } 10 \mathrm{hr}}$ and $\kappa$ (for those GRBs for which this is possible) using the values of the microphysical parameters inferred from the fits to the broadband afterglow data that were performed by Panaitescu \& Kumar (2002, denoted by PK02, using Table 2 therein) and by Yost et al. (2003, denoted by Y03, using Table 1 therein). Finally, we compare the values we obtain for $E_{\mathrm{k}, \text { iso,10hr }}$ using equation (5) to those obtained for $E_{\mathrm{k}, \text { iso }}(1$ day $)=E_{\mathrm{k}, \text { iso }, 1 \mathrm{~d}}$ by Y03 and those obtained for $E_{\mathrm{k}, \text { iso }}(10 \mathrm{hr}) \approx 0.5 E_{\mathrm{k}, \text { iso }, 0}$ by PK02. Also shown in Table 1 are the corresponding values of $\kappa$, including $\kappa_{1 \mathrm{~d}}=E_{\gamma, \text { iso }} / E_{\mathrm{k}, \text { iso }, 1 \mathrm{~d}}^{Y 03}$.

When using the fiducial parameters $\epsilon_{e}=0.1, \epsilon_{B}=0.01$, and $p=2.2$ our estimates for $E_{\mathrm{k}, \text { iso, } 10 \mathrm{hr}}$ are significantly larger than the estimates of Lloyd-Ronning \& Zhang (2004), who use the same values. This can be traced to the difference in the numerical factor that appears in equation (5). These relatively large values of $E_{\mathrm{k}, \text { iso, } 10 \mathrm{hr}}$ lead to a typical value of $\kappa$ around $0.1-0.2$, for which the $\gamma$-ray efficiency problem would not be very severe. Similar results were obtained by Fan \& Piran (2006), whose estimates for $E_{\mathrm{k}, \text { iso, } 10 \mathrm{hr}}$ are within a factor of 2 of those presented here.

The situation is different when we use the values of the microphysical parameters from the pre-Swift afterglow fits. In these cases the values of $E_{\mathrm{k}, \text { iso, } 10 \mathrm{hr}}$ are typically lower, resulting in higher estimates for $\kappa$. The estimates of the kinetic energy from the PK02 fits to the afterglow data (rather than from using eq. [5]) are generally the lowest. ${ }^{5}$ The corresponding values of $\kappa$ are close to unity. These results reflect the pre-Swift inference that there exists a rough equality between the isotropic-equivalent $\gamma$-ray and late $(10 \mathrm{hr})$ kinetic energies. The comparison between the detailed calculations and those based on equation (5) may suggest that we might be better off adopting different fiducial parameters (e.g., $\epsilon_{e}=0.3, \epsilon_{B}=0.08$, $p=2.2$ ). We also note that the values of $E_{\mathrm{k}, \text { iso, } 10 \mathrm{hr}}$ obtained by substituting the values of the microphysical parameters from the afterglow fits into equation (5) are generally higher (corresponding to lower values of $\kappa$ ) compared to those derived directly by those fits. The "typical" values of the microphysical parameters inferred from the fits are roughly $\epsilon_{e} \approx 0.3$, $\epsilon_{B} \approx 0.03, p \approx 2.2$.

The values of $\kappa$ obtained here are crucial to the overall estimate of $\epsilon_{\gamma}$ and to the origin of the "high efficiency problem." Considering Table 1, one should proceed with care in view

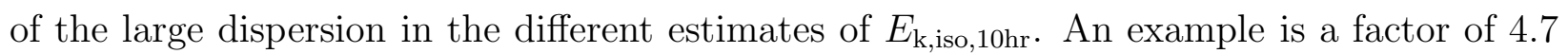
between the independent estimates of PK02 and of Y03 for GRB 000926 (and a factor of 2.5 in the opposite direction for GRB 970508). The dispersion is even greater between the

\footnotetext{
${ }^{5}$ In PK02 the values of $E_{\mathrm{k}, \text { iso }}(10 \mathrm{hr})$ are typically a factor of $\sim 2$ smaller than $E_{\mathrm{k}, \text { iso, } 0}$ due to radiative losses at early times (A. Panaitescu, personal communication; no energy injection is assumed in that work).
} 
estimates of $E_{\mathrm{k}, \text { iso }}$ from the afterglow fits and the values obtained using equation (5) for the same values of the microphysical parameters - a factor of 18 for Y03 (and 11 for PK02) for GRB 000926 .

\subsection{Swift GRBs}

The new result found by Swift is the appearance of a rapid decay followed by a shallow decline phase in the X-ray afterglow. For seven out of the ten Swift GRBs considered here there was a clear observation of the two breaks in the light curve, at $t_{\text {break, } 1}$ and $t_{\text {break,2 }}$ (the beginning and end, respectively, of the flattish segment of the X-ray light curve). If we interpret the shallow decline as arising from an additional injection of energy into the afterglow shock (see $\S 1$ ) then the ratio of the X-ray fluxes at the end and at the beginning of this phase can be used to estimate $f$ (the corresponding ratio of the kinetic energies) for these bursts (Nousek et al. 2005). A lower limit, $f_{\min }$, is obtained using the flux decrement at $t_{\text {break,1 }}$ relative to the extrapolation back to that time of the late-time $\left(t>t_{\text {break }, 2}\right)$ flux. An approximate upper limit, $f_{\max }$, is obtained by assuming that the flat part of the emission from the forward shock starts at $t_{\mathrm{dec}} \sim T_{\mathrm{GRB}}$ and is simply buried underneath the tail of

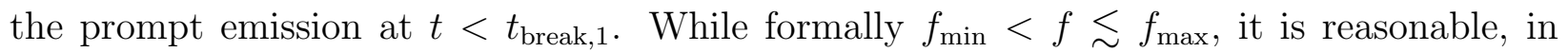
the context of the basic jet model, that the assumption made to calculate $f_{\max }$ is basically applicable, so that $f \sim f_{\max }$. Under this assumption one infers $f \gtrsim 10$, and in some cases even a much larger value of $f\left(\sim 10^{2}-10^{3}\right)$.

The results for $f$ need to be combined with an estimate of $\kappa$. Table 2 shows the values of $E_{\mathrm{k}, \text { iso, } 10 \mathrm{hr}}$ and $\kappa$ for the ten Swift GRBs with known redshifts from the Nousek et al. (2005) sample, estimated using equation (5) with $\epsilon_{e}=0.1$ and $\epsilon_{B}=0.01$. The value of $p$ was derived using the measured spectral slope in the X-ray band (attempting to fit it into the range $2<p<3$ if allowed within the measurement errors). Lacking any broad-band fits to Swift bursts, this is the best direct evidence that we have so far from these data. The resulting values of $\kappa$ are similar to those from the pre-Swift era (see Table 1). With the exception of GRB 050401, for which $\kappa=0.41$, and a few other bursts for which we only have upper limits, the inferred values of $\kappa$ are less than 0.1. If this is the correct value of $\kappa$ then, using

$$
\epsilon_{\gamma}=\left(1+\frac{\epsilon_{\mathrm{obs}, \mathrm{GRB}}}{\kappa f}\right)^{-1}
$$

(see eq. [3]), we find that with $f \sim 10$ the overall $\gamma$-ray efficiency is not larger than $\sim 0.5$ (assuming $\epsilon_{\mathrm{obs}, \mathrm{GRB}} \sim 1$ ). A similar conclusion was reached by Fan \& Piran (2006).

One may question this conclusion in view of the fact that, in pre-Swift bursts, broad- 
band analyses of the afterglow data generally resulted in a significantly lower values of $E_{\mathrm{k}, \text { iso, } 10 \mathrm{hr}}$, and correspondingly higher values of $\kappa$, compared to those obtained from equation (5) with the same fiducial values of the microphysical parameters $\left(\epsilon_{e}=0.1\right.$ and $\epsilon_{B}=0.01$; see Table 1). Furthermore, the choice of the fiducial values of the microphysical parameters is somewhat arbitrary, and it affects the resulting values of $E_{\mathrm{k}, \text { iso,10hr }}$ and $\kappa$. It is reasonable to expect that the values of the microphysical parameters that would have been inferred from a broad-band fit to the afterglow data of the Swift bursts would have led to higher

estimates of $E_{\mathrm{k}, \text { iso,10hr }}$ and $\kappa$ that were similar to those derived for the pre-Swift GRBs. The latter values, however, vary among the different estimates, from as high as $\sim 5-8$ to as low as $\sim 0.1-0.3$ (see Table 1 ). In light of this, one may adopt a "typical" value of $\kappa \sim 1$, keeping in mind that there is an uncertainty of almost an order of magnitude in this value.

The adoption of this higher value of $\kappa(\sim 1)$ for the Swift GRBs, similar to the values inferred from broad-band modeling of pre-Swift sources, together with the interpretation of the shallow decay phase as arising from energy injection (and hence $f \sim 10$ ) leads to the conclusion that typically $\epsilon_{\gamma} \sim 0.9$, and in some cases $\epsilon_{\gamma}$ is even as high as $\sim 99 \%$ (or, equivalently, $1-\epsilon_{\gamma} \approx \epsilon_{\mathrm{obs}, \mathrm{GRB}} / \kappa f$ is as low as $\left.\sim 10^{-3}-10^{-2}\right)$. Such a high $\gamma$-ray efficiency would be extremely hard to produce in the internal shocks model (see $\S 1$ ).

If, on the other hand, $\kappa \sim 0.1$ and there is energy injection (i.e. $f \sim 10$ ), or if $\kappa \sim 1$ and there is no energy injection (i.e. $f=1$; see $\S 3$ ), then this would imply a significantly lower typical $\gamma$-ray efficiency, $\epsilon_{\gamma} \sim 0.5$, although in some cases $\epsilon_{\gamma}$ would still be as high as $\sim 90 \%$ (or, equivalently, $1-\epsilon_{\gamma} \approx \epsilon_{\mathrm{obs}, \mathrm{GRB}} / \kappa f$ would still be as low as $\sim 10^{-2}-10^{-1}$ ). Even the latter, more moderate, requirements on the $\gamma$-ray efficiency are not easily satisfied in the internal-shocks model, athough they might possibly still be accommodated in this scenario (Kobayashi et al. 1997; Kumar 1999; Guetta et al. 2001). Finally, if $\kappa \sim 0.1$ and the shallow decline does not arise from energy injection (i.e., $f=1$ ) but, say, from varying afterglow efficiency (as discussed in $\S 3$ ), then the $\gamma$-ray efficiency would typically be $\epsilon_{\gamma} \sim 0.1$. In the latter case the results are fully consistent with the predictions of the internal-shocks model.

\subsection{Some Caveats}

The discussion so far was relevant to the power-law segment of the spectrum where $\nu_{\mathrm{X}}>\max \left(\nu_{m}, \nu_{c}\right)$. If, instead, $\nu_{m}<\nu_{\mathrm{X}}<\nu_{c}$, we have only a lower limit on the value of $E_{\mathrm{k} \text {,iso }}$ from this consideration (i.e., from eq. [5]) that corresponds to an upper limit on the value of $\kappa$, the true value being smaller than this upper bound by a factor $\left[\nu_{c}(10 \mathrm{hr}) / \nu_{\mathrm{X}}\right]^{2 /(p+2)}$ (which, however, is not typically expected to be very large). 
It is possible that there is a contribution to $F_{\mathrm{X}}\left(t_{\mathrm{dec}}\right)$ from a component of the outflow that is not along the line of sight, or for some other reason did not contribute to the observed $\gamma$-ray emission. In this case equations (3) and (5) would provide a lower limit on $\epsilon_{\gamma}$, rather than directly determine its value. If both the prompt $\gamma$-ray emission and $F_{\mathrm{X}}\left(t_{\mathrm{dec}}\right)$ were dominated by emission from angles $\theta>1 / \Gamma$ relative to the line of sight (where $\Gamma$ is the Lorentz factor of the outflow), then again equations (3) and (5) would provide a lower limit on $\epsilon_{\gamma}$, since the beaming of radiation away from the line of sight is expected to be either comparable or somewhat smaller during the afterglow emission at $t_{\mathrm{dec}}$ compared to the prompt GRB.

It is also possible that some fraction $\epsilon_{*}$ of the original energy $E_{\text {iso }, 0}$ ended up in a totally different form, i.e., was not radiated during the prompt emission and did not end up in the kinetic energy of the afterglow shock. This could occur, for example, if along some directions a forward shock is not formed (or at least not formed efficiently) for a very pure Poyntingflux outflow. In such a case some of the energy (potentially even most of the energy) could be carried out to very large distances (in principle out to infinity) in electromagnetic form (such as low-frequency electromagnetic waves). ${ }^{6}$ Alternatively, a good fraction of the energy might be carried away in high-energy cosmic rays and neutrinos (Waxman 1995; Waxman \& Bahcall 1997) and thus would not contribute to the kinetic energy of the afterglow shock. In this case we have $E_{\mathrm{k}, \text { iso }, 0}=\left(1-\epsilon_{\gamma}-\epsilon_{*}\right) E_{\mathrm{iso}, 0}$ and we need to make the substitution $\left(1-\epsilon_{\gamma}\right) \rightarrow\left(1-\epsilon_{\gamma}-\epsilon_{*}\right)$ everywhere, so the estimate (13) for $\epsilon_{\gamma}$ (assuming $\epsilon_{*}=0$ ) should be multiplied by $\left(1-\epsilon_{*}\right)$,

$$
\epsilon_{\gamma}=\left(1-\epsilon_{*}\right)\left(1+\frac{\epsilon_{\mathrm{obs}, \mathrm{GRB}}}{\kappa f}\right)^{-1} .
$$

This means that $\epsilon_{\gamma}<1-\epsilon_{*}$ (even for $\kappa f \gg \epsilon_{\mathrm{obs}, \mathrm{GRB}}$ ), and therefore $\epsilon_{*} \gtrsim 0.5$ would imply $\epsilon_{\gamma} \lesssim 0.5$. Thus, even when one infers $\epsilon_{\gamma} /\left(1-\epsilon_{\gamma}\right) \gg 1$ and hence $1-\epsilon_{\gamma} \ll 1$ under the usual assumption that $\epsilon_{*}=0$ (or at least $\epsilon_{*} \ll 1-\epsilon_{\gamma}$ ), the intrinsic $\gamma$-ray efficiency might still be significantly smaller, and is $\epsilon_{\gamma} \lesssim 0.5$ for $\epsilon_{*} \gtrsim 0.5$. Note that, in order for GRBs to produce the highest-energy cosmic rays, their energy should be comparable to that of the prompt $\gamma$-rays (Waxman 1995, 2004), i.e., $\epsilon_{\gamma} \lesssim \epsilon_{*}$ (the inequality arising since there might be other forms of energy that escape the prompt emission site without being directly detected), and therefore $\epsilon_{\gamma} \lesssim 0.5$.

One should, however, keep in mind that high-energy cosmic rays and neutrinos must tap the same dissipated energy that also powers the prompt $\gamma$-ray emission. Therefore, for the same observed energy in $\gamma$-rays and inferred kinetic energy in the afterglow, in addition to increasing the required total initial energy $E_{\mathrm{iso}, 0}$ by a factor of $\left(1-\epsilon_{*}\right)^{-1}$, these particles

\footnotetext{
${ }^{6}$ Both the formation of a forward shock and the ability of energy to escape to infinity in electromagnetic form have not yet been fully worked out (e.g., Melatos \& Melrose 1996; Smolsky \& Usov 2000).
} 
would also increase the required dissipated energy $\epsilon_{\mathrm{dis}, \mathrm{GRB}} E_{\mathrm{iso}, 0}$ and the fraction $\epsilon_{\mathrm{dis}, \mathrm{GRB}}$ of the dissipated energy that ends up in $\gamma$-rays. ${ }^{7}$ In other words, $\epsilon_{\text {dis,GRB }} \geq \epsilon_{\gamma}+\epsilon_{*}=$ $\left(E_{\gamma, \text { iso }}+E_{* \text {,iso }}\right) /\left(E_{\mathrm{k}, \text { iso }, 0}+E_{\gamma, \text { iso }}+E_{* \text {,iso }}\right)>E_{\gamma, \text { iso }} /\left(E_{\mathrm{k}, \text { iso }, 0}+E_{\gamma, \text { iso }}\right)$, where $E_{* \text {,iso }}=\epsilon_{*} E_{\text {iso }, 0}$ is the (isotropic-equivalent) energy in cosmic rays and neutrinos, and $E_{\mathrm{k}, \text { iso, } 0}$ and $E_{\gamma, \text { iso }}$ are determined (at least in principle) by observations. Clearly, $E_{*, \text { iso }}$ increases the lower limit on $\epsilon_{\text {dis,GRB }}$. Nevertheless, the fact that $E_{* \text {,iso }}$ may reduce $\epsilon_{\gamma}$ to $\lesssim 0.5$ even for $\kappa f \gg 1$ makes it possible to have $\epsilon_{e, \mathrm{GRB}} \lesssim 0.5$, which should be easier to accommodate for shock acceleration in a proton-electron plasma. Still, dissipating and getting rid of almost all of the energy (through radiation, cosmic rays, neutrinos, etc.) and leaving only a small fraction of the original energy in the kinetic energy of the forward shock, as is required for $\kappa f \gg 1$, is not an easy task for any model of the prompt emission.

\section{Efficiency of the X-Ray Afterglow Emission}

As noted in $\S 1$, one of the new features discovered by Swift is the early shallow decline phase: $F_{\mathrm{X}} \propto t^{-\alpha}$ with $0.2 \lesssim \alpha \lesssim 0.8$ for $t_{\text {break }, 1} \lesssim t \lesssim t_{\text {break }, 2}$. During this phase $t F_{\mathrm{X}}(t)$ increases with time. Using the definition of $\epsilon_{\mathrm{X}}(t)$ (eq. [2]) and the relation between $L_{\mathrm{X} \text {,iso }}$ and $F_{\mathrm{X}}$ (eq. [1]), we find that

$$
\frac{\epsilon_{\mathrm{X}}(t) E_{\mathrm{k}, \text { iso }}(t)}{t F_{\mathrm{X}}(t)}=4 \pi d_{L}^{2}(1+z)^{\beta-\alpha-1}
$$

is constant in time. Note that if $\nu$ and $t$ in the expression for $F_{\mathrm{X}}(t)$ were referred to the GRB rest frame rather than to the observer frame then the factor $(1+z)^{\beta-\alpha-1}$ on the right-hand side of equation (15) would be eliminated, and with it any potential (weak) time dependence resulting from a possible temporal variation of $\alpha$ or $\beta$.

Now, if the observed frequencies satisfy $\nu_{\mathrm{X}}>\max \left(\nu_{m}, \nu_{c}\right)$, and $p>2$, then equation (4) is applicable. Furthermore, if the afterglow shock evolves according to the adiabatic selfsimilar solution of Blandford \& McKee (1976) and the fractions of the postshock internal energy in electrons $\left(\epsilon_{e}\right)$ and in magnetic field $\left(\epsilon_{B}\right)$ are constant in time, then $\nu_{m} \propto t^{-3 / 2}$. Under these circumstances $\epsilon_{\mathrm{X}} \propto t^{-3(p-2) / 4}$ decreases (slowly) with time. As can be seen from equation (15), $\epsilon_{\mathrm{X}}(t) E_{\mathrm{k}, \text { iso }}(t) \propto t F_{\mathrm{X}}(t)$. Therefore, the observed rise in $t F_{\mathrm{X}}(t)$ implies a similar rise in $\epsilon_{\mathrm{X}}(t) E_{\mathrm{k} \text {,iso }}(t)$. Given the expected decrease of $\epsilon_{X}(t)$ with $t$ for $p>2$, the slowly decaying portion of the light curve has been attributed by several researchers to an increase

\footnotetext{
${ }^{7}$ In the case of a very pure Poynting flux, the escaping energy $E_{*, \text { iso }}$ is the fraction that did not dissipate. Therefore, while $E_{\mathrm{iso}, 0}$ increases by a factor of $\left(1-\epsilon_{*}\right)^{-1}$, the dissipated energy $\epsilon_{\mathrm{dis}, \mathrm{GRB}} E_{\mathrm{iso}, 0}$ remains unchanged (assuming other efficiencies do not change), and thus $\epsilon_{\text {dis, GRB }}$ decreases by a factor of $\left(1-\epsilon_{*}\right)^{-1}$.
} 
in $E_{\mathrm{k} \text {,iso }}(t)$, i.e., to some sort of energy injection into the forward shock (e.g., Nousek et al. 2005; Panaitescu et al. 2005; Zhang et al. 2005; Granot \& Kumar 2006).

It is, however, conceivable that the rise in $t F_{\mathrm{X}}(t)$ corresponds, at least in part, to an increase of $\epsilon_{\mathrm{X}}(t)$ with time. One way in which this could be brought about is if $p$ were $<2$ (assuming $\epsilon_{e}$ and $\epsilon_{B}$ remain constant). ${ }^{8}$ In this case $N\left(\gamma_{e}\right) \propto \gamma_{e}^{-p}$ for $\gamma_{\min }<\gamma_{e}<\gamma_{\max }$ and $\epsilon_{\mathrm{X}} \sim(1+Y)^{-1} \epsilon_{e}\left(\nu_{\max } / \nu_{\mathrm{X}}\right)^{(p-2) / 2}$ [the same as eq. (4) for $p<2$, but with $\nu_{\max }$ replacing $\nu_{m}$ ], where $\nu_{\max }=\nu_{\text {syn }}\left(\gamma_{\max }\right) \propto \gamma B \gamma_{\max }^{2} \propto \gamma^{4} \rho_{\text {ext }}^{1 / 2} \propto t^{-3 / 2}$ [where $B$ is the comoving magnetic field amplitude and $\rho_{\text {ext }}$ is the external density; the same as the scaling of $\nu_{m}=\nu_{\text {syn }}\left(\gamma_{\min }\right)$ for $p>2$ ], so $\epsilon_{\mathrm{X}} \propto t^{3(2-p) / 4}$. This time dependence is the same as for $p>2$, but in this case $\epsilon_{\mathrm{X}}$ increases with time whereas for $p>2$ it decreases. Similarly, $F_{\mathrm{X}} \propto t^{-(3 p-2) / 4}$, just as for $p>2$, except that for $p<2$ this corresponds to a decay rate flatter than $t^{-1}$. One possible difference between the two cases is that for $p<2$ and $\nu_{c}<\nu_{\max }$ radiative losses are not always negligible since most of the energy in the electrons is at $\gamma_{e} \sim \gamma_{\max }$ and is therefore radiated away. Thus, unless $\epsilon_{e} \ll 1$, radiative losses could be significant and would tend to steepen the flux decay rate and make it harder to achieve a flattish light curve. We also note that the X-ray spectral slope for $p<2$ [assuming $\nu_{\mathrm{X}}>\max \left(\nu_{c}, \nu_{m}\right)$ ] is $\beta_{\mathrm{X}}=p / 2<1$, which in many cases is inconsistent with the observed value (Nousek et al. 2005), so this explanation of the shallow decay of $F_{\mathrm{X}}$ might only apply to a subset of the sources (see Fig. 8 of Nousek et al. 2005).

An alternative possibility for $\epsilon_{\mathrm{X}}(t)$ to increase with $t$ is for either one (or both) of the microphysical parameters $\epsilon_{e}$ and $\epsilon_{B}$ to increase with time. Using the dependence of $\nu_{m}$ on these parameters (e.g., Sari et al. 1998), we find that, for $p>2, \epsilon_{\mathrm{X}} \propto \epsilon_{e}^{(p-1)} \epsilon_{B}^{(p-2) / 4}$ when $\epsilon_{e} \ll \epsilon_{B}$ and $\epsilon_{\mathrm{X}} \propto \epsilon_{e}^{(p-3 / 2)} \epsilon_{B}^{p / 4}$ when $\epsilon_{e} \gg \epsilon_{B}$. This applies when parameterizing in terms of $E_{\mathrm{k} \text {,iso }}$ (which is not measured directly), whereas a parameterization in terms of $L_{\mathrm{X} \text {,iso }}$ (which is measured directly) yields $\epsilon_{\mathrm{X}} \propto \epsilon_{e}^{4(p-1) /(p+2)} \epsilon_{B}^{(p-2) /(p+2)}$ when $\epsilon_{e} \ll \epsilon_{B}$ and $\epsilon_{\mathrm{X}} \propto \epsilon_{e}^{2(2 p-3) /(p+2)} \epsilon_{B}^{p /(p+2)}$ when $\epsilon_{e} \gg \epsilon_{B}$ (see eq. [2]). Table 3 of Nousek et al. (2005) provides the values of $\Delta \alpha$ - the change in the temporal decay index across the break in the light curve at $t_{\text {break,2 }}$ - for nine Swift GRBs in which it could be measured reliably, and shows that typically $0.5 \lesssim \Delta \alpha \lesssim 1$. In our context, if $\epsilon_{e} \propto t^{\alpha_{e}}$ and $\epsilon_{B} \propto t^{\alpha_{B}}$ at $t<t_{\text {break,2 }}$, then attributing the flattish decay phase to a growth of $\epsilon_{e}$ and/or $\epsilon_{B}$ with time requires (in the limit $\left.\epsilon_{e} \gg \epsilon_{B}\right)$ that $\Delta \alpha=2(2 p-3) /(p+2) \alpha_{e}+\alpha_{B} p /(p+2)$. For $2<p<3,0.5<p /(p+2)<0.6$ and $0.5<2(2 p-3) /(p+2)<1.2$. Therefore, for $p \approx 2, \alpha_{e}+\alpha_{B} \approx 2 \Delta \alpha \sim 1-2$ and

${ }^{8} \mathrm{It}$ is in principle possible that $\epsilon_{\mathrm{X}}$ could increase with time on account of its dependence on $Y$ (see eq. [4) even if $\epsilon_{e}$ and $\epsilon_{B}$ remained constant and $p$ were $>2$, given that $Y$ decreases with time in the slowcooling regime $\left(\nu_{m}<\nu_{c}\right)$ that is relevant for $p>2$. However, one can show that, to be relevant during the early-afterglow phase, this would require unrealistically high values of $p$. 
for $\Delta \alpha \sim 0.5$, a roughly linear growth with time of either $\epsilon_{e}$ or $\epsilon_{B}$ (or of their product) is required. For $p \sim 2.6$ and $\Delta \alpha \sim 1$, a linear growth of $\epsilon_{e}$ and a constant $\epsilon_{B}$ would work (i.e. $\alpha_{e} \approx 1$ and $\left.\alpha_{B}=0\right)$. If $Y \sim 1$ (rather than $Y \gg 1$ or $Y \ll 1$ ), the dependence of $\epsilon_{\mathrm{X}}$ on $\epsilon_{e}$ and $\epsilon_{B}$ is no longer a simple power law, requiring a similarly nontrivial dependence of $\epsilon_{e}$ and/or $\epsilon_{B}$ on the observed time $t$ (insofar as $F_{\mathrm{X}}$ is indeed a pure power law in $t$ during the flattish decay phase). A physical scenario will need to account both for this behavior and for why the time evolution of the microphysical parameters effectively terminates at $t_{\mathrm{break}, 2}$.

The magnetic-energy parameter $\epsilon_{B}$ could reflect either the structure of the ambient magnetic field or postshock field-amplification processes. In the former case an increase of $\epsilon_{B}$ with time could be caused by an increase of the magnetization parameter $\sigma=B_{\text {ext }}^{2} / 4 \pi \rho_{\text {ext }} c^{2}$ of the ambient gas with distance from the source, which might occur in certain GRB progenitor models (e.g., Königl \& Granot 2002). In the latter case one cannot at present identify a natural reason for $\epsilon_{B}$ to increase during the early afterglow phase, but future theoretical advances (see, e.g., Medvedev et al. 2005) might make it possible to study the evolution of shock-generated magnetic fields over time scales that are long enough to address this question. The value of $\epsilon_{e}$ might also be linked to the changing shock parameters (in particular, the shock Lorentz factor). However, in this case, again, our current level of understanding does not allow us to make a specific prediction.

The possible increase of the afterglow radiation efficiency with time during the early phases of the X-ray light curve may also help to lower the estimate of the $\gamma$-ray radiative efficiency and thereby alleviate the constraints on the internal-shocks model. If at early times $\left(t<t_{*}\right) \epsilon_{\mathrm{X}}$ increased with $t$, then (by eq. [15]) $E_{\mathrm{k}, \text { iso, } 0}$ would be underestimated, and therefore the parameter $f$ and the value of $\epsilon_{\gamma}$ would be overestimated. The prompt emission from internal shocks could in principle be observable even if the very early radiation from the external (forward) shock is weak because of a low value of $\epsilon_{B}$ or of a possible suppression of $\epsilon_{e}$ when the shock Lorentz factor is high. This is because the value of $\epsilon_{B}$ in the internal shocks might be determined by a comparatively strong magnetic field advected from the central source (e.g., Spruit et al. 2001) and because (in contrast to the forward shock at the deceleration time, whose Lorentz factor is $\gtrsim 10^{2}$ ) the internal shocks are only mildly relativistic. If the afterglow emission efficiency recovers its commonly assumed value (equation [4]) at $t \gtrsim t_{\text {break,2 }}$ then one could in principle have $f \sim 1, \kappa \sim 1$, and $\epsilon_{\gamma} \sim 0.5$. In this case $E_{\mathrm{k}, \text { iso }}$ remains constant while the flattish flux decay is caused by an increase of $\epsilon_{X}$ with time. However, if even at late times the afterglow efficiency is only a fraction $\delta \ll 1$ of its standard value [where $\delta$ might, for example, correspond to the fraction $\xi_{e}$ of the electrons behind the forward shock that are accelerated to relativistic energies, which could conceivably be $\ll 1$ (Eichler \& Waxman 2005)] then $\kappa \sim \delta \kappa_{\text {standard }} \sim \delta \ll 1$ and $\epsilon_{\gamma} \sim \delta$ (for $\left.\epsilon_{\mathrm{obs}, \mathrm{GRB}} \sim 1\right)$. In this case $E_{\mathrm{k}, \text { iso }}$ is again constant with time and the flattish decay phase is 
due to $\epsilon_{X}$ increasing with time; however, the value of $\epsilon_{X}$ at late times is $\delta$ times its standard value (given by equation [4]) and the true kinetic energy in the afterglow shock is a factor $\delta^{-1} \gg 1$ larger than the usual estimate of $\sim 10^{51} \mathrm{erg}$.

An alternative to the explanation of the flattish segment of the light curve in terms of a prolonged energy injection into the forward shock of a basic jet model is the possibility (in a generalized jet model) of distinct spatial components, some of which only contribute to the afterglow emission at later times. This situation could arise if (1) some of the ejecta have lower initial Lorentz factors that result in longer deceleration times, so only a small fraction of the injected energy is transferred to the shocked external medium early on, or if (2) radiation from components that do not move along our line of sight is strongly beamed away from us at early times, becoming visible only later on when deceleration causes the respective beaming cones to widen. Case (1) is exemplified by the two-component jet model considered in the next section, whereas case (2) might be realized in the "patchy shell" model of GRB sources (Kumar \& Piran 2000) and in the "anisotropic afterglow efficiency" scenario outlined by Eichler \& Granot (2005). ${ }^{9}$

\section{The Two-Component Jet Model: A Case Study}

As pointed out in $\S 1$, the two-component jet model was originally invoked by Peng et al. (2005) as a possible way of alleviating the pre-Swift constraints on the $\gamma$-ray emission efficiency. In this section we interpret the Swift results in the context of this scenario, using again the parameters $\kappa$ and $f$ introduced in $\S 2$ and affirming some of the conclusions reached in that section. In this discussion we assume that the standard afterglow theory applies and that the microphysical parameters do not change with time. This formulation is used to demonstrate that the recent observations are inconsistent with parameter values of the twocomponent jet model that could lead to a lower inferred magnitude for $\epsilon_{\gamma}$, reinforcing the result that, in the context of the standard afterglow theory, the Swift measurements have tightened the constraints on the prompt-emission efficiency. We go on to show, however, that the two-component model can nevertheless account for the X-ray afterglow light curve of GRB sources, including the flattish early-time segment.

The generic two-component jet model consists of a narrow and initially highly relativistic (conical) outflow from which the prompt emission originates, and a moderately relativistic flow that decelerates at a significantly later time and occupies a wider (coaxial) cone. The

\footnotetext{
${ }^{9}$ Case (2) might also be relevant to the two-component jet model if the line of sight to the observer lies within the solid angle subtended by the narrow component but outside the inner edge of the wide component.
} 
narrow component is the source of the prompt emission (which is observed as a GRB if the observer's line of sight lies within, or very close to, its opening solid angle), whereas the wide component only makes a contribution to the afterglow emission (which becomes observable after it decelerates). Letting $E_{i}, E_{\mathrm{k}, i}, \theta_{i}$, and $\eta_{i}$ stand for the total initial energy, initial kinetic energy, opening half-angle, and Lorentz factor of the two components (with $i=\mathrm{n}, \mathrm{w}$ corresponding to narrow and wide, respectively), we have

$$
E_{\gamma, \text { iso }}=\epsilon_{\gamma} E_{\mathrm{n}, \text { iso }}=\epsilon_{\gamma} E_{\mathrm{n}} \frac{2}{\theta_{\mathrm{n}}^{2}} .
$$

The parameter $\kappa$ defined in equation (3) can be expressed in this context by the relation

$$
E_{\gamma, \text { iso }}^{\mathrm{obs}} \frac{\theta_{\mathrm{w}}^{2}}{2}=\epsilon_{\mathrm{obs}, \mathrm{GRB}} \epsilon_{\gamma} E_{\mathrm{n}}\left(\frac{\theta_{\mathrm{w}}}{\theta_{\mathrm{n}}}\right)^{2} \approx \kappa\left(E_{\mathrm{k}, \mathrm{n}}+E_{\mathrm{k}, \mathrm{w}}\right),
$$

where $E_{\mathrm{k}, \mathrm{n}}=\left(1-\epsilon_{\gamma}\right) E_{\mathrm{n}}$ and $E_{\mathrm{k}, \mathrm{w}} \approx E_{\mathrm{w}}$. Equation (17) incorporates the fact that the kinetic energy responsible for the late afterglow emission is determined empirically by assuming a jet of half-opening angle $\theta_{\mathrm{w}}$, or equivalently that the isotropic-equivalent kinetic energy inferred from late time afterglow corresponds approximately to the total kinetic energy over the fraction of the total solid angle occupied by the wide component.

The energy that determines the early afterglow phase is

$$
E_{\text {early,iso }}=E_{\mathrm{k}, \mathrm{n}} \frac{2}{\theta_{\mathrm{n}}^{2}}=E_{\mathrm{k}, \mathrm{n}, \text { iso }}
$$

whereas the late afterglow phase is determined by

$$
E_{\text {late, iso }}=\left(E_{\mathrm{k}, \mathrm{n}}+E_{\mathrm{k}, \mathrm{w}}\right) \frac{2}{\theta_{\mathrm{w}}^{2}} .
$$

The evidence from the Swift observations that we do not see the early afterglow emission above the rapidly decaying tail of the prompt emission, and that even when it shows up it is rather weak, implies that the early isotropic kinetic energy $E_{\text {early, iso }}$ is a factor $f \sim 10$ smaller than the late isotropic kinetic energy $E_{\text {late,iso }}$, where we reintroduced the parameter $f$ defined in equation (3). This implies that

$$
\frac{E_{\mathrm{k}, \mathrm{n}}}{\theta_{\mathrm{n}}^{2}}=\frac{E_{\mathrm{k}, \mathrm{n}}+E_{\mathrm{k}, \mathrm{w}}}{f \theta_{\mathrm{w}}^{2}} \approx \frac{E_{\mathrm{k}, \mathrm{w}}}{f \theta_{\mathrm{w}}^{2}}
$$

which in turn implies that

$$
E_{\mathrm{k}, \mathrm{n}}<E_{\mathrm{k}, \mathrm{w}} .
$$

Hence we can delete the term involving $E_{\mathrm{k}, \mathrm{n}}$ from the right-hand side of equation (17) and obtain

$$
\frac{\epsilon_{\gamma} \epsilon_{\mathrm{obs}, \mathrm{GRB}}}{\left(1-\epsilon_{\gamma}\right)}=\kappa \frac{E_{\mathrm{k}, \mathrm{w}}}{E_{\mathrm{k}, \mathrm{n}}}\left(\frac{\theta_{\mathrm{n}}}{\theta_{\mathrm{w}}}\right)^{2}=\kappa \frac{E_{\mathrm{k}, \mathrm{w}, \text { iso }}}{E_{\mathrm{k}, \mathrm{n}, \text { iso }}}
$$


Expressing now $E_{\mathrm{k}, \mathrm{w}}$ in terms of $E_{\mathrm{k}, \mathrm{n}}$ using this last equation and substituting into equation (20) (which becomes simply $f \approx E_{\mathrm{k}, \mathrm{w}, \text { iso }} / E_{\mathrm{k}, \mathrm{n} \text {,iso }}$ when one neglects $E_{\mathrm{k}, \mathrm{n}}$ on the r.h.s.), we rediscover the first relation in equation (3), which can be expressed in the form of equation (13) to yield $\epsilon_{\gamma} \sim 0.9$ for $f \sim 10$ and $\left(\kappa / \epsilon_{\mathrm{obs}, \mathrm{GRB}}\right) \sim 1$.

Equation (22) with $\epsilon_{\gamma} \epsilon_{\mathrm{obs}, \mathrm{GRB}} \approx 1$ implies that $\kappa E_{\mathrm{k}, \mathrm{w}} / \theta_{\mathrm{w}}^{2} \approx E_{\mathrm{n}} / \theta_{\mathrm{n}}^{2}$, and hence, given that $E_{\mathrm{k}, \mathrm{w}} \approx E_{\mathrm{w}}$ and taking $\kappa$ to be $\sim 1$, that the narrow and wide jet components initially have comparable isotropic-equivalent energies. The ratio of true energies of the two components is initially $E_{\mathrm{w}} / E_{\mathrm{n}} \approx \kappa^{-1}\left(\theta_{\mathrm{w}} / \theta_{\mathrm{n}}\right)^{2} \sim(4-9) \kappa^{-1}$ for reasonable ratios of the opening halfangles. This ratio further increases by a factor $\left(1-\epsilon_{\gamma}\right)^{-1} \approx \kappa f$ (see eq. [13]) during the prompt-emission phase.

As argued by Peng et al. (2005), this model could reduce the inferred value of $\epsilon_{\gamma}$ if $E_{\mathrm{k}, \mathrm{w}} / E_{\mathrm{k}, \mathrm{n}}>1$ and $E_{\mathrm{k}, \mathrm{w}, \text { iso }} / E_{\mathrm{k}, \mathrm{n}, \text { iso }}<1$. However, the Swift results, as expressed by equation (20), demonstrate that the latter ratio is equal to $f \sim 10$, and hence that this possibility is not realized in practice. Peng et al. (2005) also discussed the ability of this scenario to account for certain "bumps" in the late-afterglow light curve of several pre-Swift GRB sources. We now show that this model can similarly account for the early-time flattening of the X-ray light curve. The required model parameters are, however, distinctly different from those considered by Peng et al. (2005); in fact, the fits that we obtain reinforce the conclusion that $E_{\mathrm{k}, \mathrm{w}, \text { iso }} / E_{\mathrm{k}, \mathrm{n}, \text { iso }}(\approx f)$ must be $\gg 1$.

The Lorentz factor of the narrow jet component does not directly enter into our modeling of the light curve, although the usual "compactness" arguments for the prompt emission (e.g., Lithwick \& Sari 2001) imply that its value is $\eta_{\mathrm{n}} \gtrsim 10^{2}$. Our interpretation of the flattish segment of the light curve in the context of this scenario is that it largely corresponds to the emergence of the wide component around its deceleration time $t_{\mathrm{dec}, \mathrm{w}} \propto\left(E_{\mathrm{k}, \mathrm{w}, \mathrm{iso}} / n_{\mathrm{ext}}\right)^{1 / 3} \eta_{\mathrm{w}}^{-8 / 3}$, where $n_{\text {ext }}$ is the particle density of the ambient medium at the deceleration radius. Owing to the strong dependence of $t_{\mathrm{dec}}$ on $\eta$, we can constrain the value of $\eta_{\mathrm{w}}$ within a factor of 2 or so.

Figure 1 shows a tentative fit to the X-ray light curve of GRB 050315 with the twocomponent jet model, whereas Figure 2 demonstrates the effect of modifying the model parameters. The extended flat segment of the light curve together with the requirement that the contribution from the narrow component at $t /(1+z) \sim 200 \mathrm{~s}$ does not overproduce the observed flux imply $f \approx E_{\mathrm{k}, \mathrm{w} \text {,iso }} / E_{\mathrm{k}, \mathrm{n}, \text { iso }} \gtrsim 30$. This suggests that $f \sim f_{\max } \sim 30$ (see Table 2) and that both $f_{\min }$ and $f_{\max }$ might have been somewhat underestimated for this event (since the fit to the data should produce $f \gtrsim f_{\min }$, suggesting that $f_{\min } \sim 30$ for GRB 050315, which is higher than the value of $f_{\min }=11$ derived by Nousek et al. 2005 and shown in Table 2). For our fiducial parameter values $\left(\epsilon_{e}=0.1, \epsilon_{B}=0.01\right.$, and $p \approx 2.0-2.1$; 
see Table 2 and Fig. 1) we find (using eq. [5]) $\kappa \approx 0.034$ (see Table 2). For this value of $\kappa$ the product $\kappa f$ is $\sim 1$, which implies (from eq. [13] with $\epsilon_{\mathrm{obs}, \mathrm{GRB}} \sim 1$ ) that $\epsilon_{\gamma} \sim 0.5$. The fit to the data shown in Figure 1 incorporates an SSC component whose contribution in this case turns out to be comparable to that of the synchrotron component at $t_{*}=10 \mathrm{hr}$, resulting in a decrease by a factor of $\sim 2$ in the estimate of $E_{\mathrm{k}, \text { iso }}(10 \mathrm{hr}$ ) (and in a corresponding increase in the estimate of $\kappa$ ) in comparison with the values inferred by using equation (5) (which only takes account of the synchrotron contribution). The actual numerical difference between these two estimates is, in fact, slightly larger (a factor $\sim 2.5$ ), reflecting the fact that the fit employs a numerical scheme that is not identical to equation (5). All in all, the model fit shown in Figure 1 yields $f \approx E_{\mathrm{k}, \mathrm{w}, \text { iso }} / E_{\mathrm{k}, \mathrm{n}, \text { iso }}=30, \kappa \approx E_{\gamma, \text { iso }}^{\text {obs }} / E_{\mathrm{k}, \mathrm{w} \text {,iso }} \approx 0.086$, and $\kappa f \approx E_{\gamma, \text { iso }}^{\mathrm{obs}} / E_{\mathrm{k}, \mathrm{n}, \mathrm{iso}} \approx 2.6$, which implies $\epsilon_{\gamma} \approx 0.72$ for $\epsilon_{\mathrm{obs}, \mathrm{GRB}}=1$.

One realization of a two-component relativistic outflow of the type considered here is an initially neutron-rich, hydromagnetically accelerated jet (Vlahakis et al. 2003). In this picture the narrow component consists of the originally injected protons that are gradually accelerated to $\eta_{\mathrm{n}}$; the injected neutrons decouple from the original proton component when the jet Lorentz factor reaches $\eta_{\mathrm{w}}$ and eventually decay to form a distinct (wide) proton component. In the illustrative solution presented in Vlahakis et al. $(2003), \eta_{w} \approx 15$. The dashed curve in Figure 2 demonstrates that, to be consistent with the data, this value of $\eta_{\mathrm{w}}$ requires a very high external density, $n_{\text {ext }} \sim 10^{3.5} \mathrm{~cm}^{-3}$ (although even for this value the fit is not as good as for the parameters adopted in Fig. 1). This can be understood from the parameter dependence of $t_{\mathrm{dec}, \mathrm{w}} /(1+z)$, for which the model fit implies a value of $\sim 2 \times 10^{3} \mathrm{~s}$. To reproduce the observed flux, the value of $E_{\mathrm{k}, \mathrm{w} \text {,iso }}$ cannot be too low. In fact, in the model fit represented by the dashed curve we have adopted the lowest possible value of this quantity, corresponding to an equipartition between the electron and magnetic field energy densities. With the values of $t_{\mathrm{dec}, \mathrm{w}}$ and $E_{\mathrm{k}, \mathrm{w} \text {,iso }}$ thus fixed, the inferred external density becomes very sensitive to the value of the Lorentz factor $\left(n_{\mathrm{ext}} \propto \eta_{\mathrm{w}}^{-8}\right)$. Intermediate options, with a somewhat higher Lorentz factor $\left(\eta_{\mathrm{w}}=21\right)$ are also shown in Figure 2, both for the uniform-density case used in the previous fits (dash-dotted curve) and for an $r^{-1.5}$ density profile (where $r$ is the distance to the source; dotted curve). We interpret the break in the light curve of GRB 050315 at $t /(1+z) \sim 1$ day as the jet break time of the wide component, $t_{\mathrm{jet}, \mathrm{w}} /(1+z)$. Since the jet break time is particularly sensitive to the value of the opening half-angle, $t_{\text {jet,w }} \propto\left(E_{\mathrm{k}, \mathrm{w}, \text { iso }} / n_{\text {ext }}\right)^{1 / 3} \theta_{\mathrm{w}}^{8 / 3}$, this allows us to constrain the value of $\theta_{\mathrm{w}}$. Given that $t_{\text {jet,w }} / t_{\mathrm{dec}, \mathrm{w}} \propto\left(\eta_{\mathrm{w}} \theta_{, \mathrm{w}}\right)^{8 / 3}$, it is seen that any reduction in the fitted value of $\eta_{\mathrm{w}}$ requires an increase in $\theta_{\mathrm{w}}$ by a similar factor.

As was already noted in Peng et al. (2005), another realization of this type of an outflow is potentially provided by a relativistic, baryon-poor jet, which is driven electromagnetically along disk-anchored magnetic field lines that thread the horizon of a rotating black hole, 
and which is "contaminated" by neutron diffusion from a baryon-rich disk wind. In the original version of this scenario, which was proposed by Levinson \& Eichler (1993, 2003; see also van Putten \& Levinson 2003) and recently studied numerically by McKinney (2005a,b), the narrow and wide components correspond to the baryon-poor and baryon-rich outflows, respectively. However, the predicted Lorentz factor of the disk wind is too low to be consistent with the rather high value of $\eta_{\mathrm{w}}$ inferred from our model fits. An alternative possibility is that the wide component corresponds to a hydromagnetically accelerated baryon-rich disk outflow of the type modeled by Vlahakis \& Königl (2003a,b), whereas the narrow component corresponds (as in the Levinson \& Eichler picture) to a baryon-poor Blandford \& Znajek (1977)-type outflow. ${ }^{10}$ It should be noted that in either one of the above two-component jet realizations the $\gamma$-ray emitting component is associated with an initially Poynting-dominated outflow. This could in principle make it possible to account for the relatively high emission efficiency that the Swift results seem to imply (see $\S 5$ ).

\section{Summary and Conclusions}

We have shown that the $\gamma$-ray efficiency implied by the Swift observations is modeldependent and can vary over a wide range (from typical values of $\epsilon_{\gamma} \sim 0.9$ or higher to $\epsilon_{\gamma} \sim 0.1$ or lower) depending on the adopted model assumptions. The $\gamma$-ray efficiency has been expressed in terms of observable quantities (see eqs. [3] and [13]), namely $\kappa$ and $f$, where $\kappa$ relates the $\gamma$-ray emission to the late-time afterglow emission (and was therefore available in the pre-Swift era) and $f$ relates the early- and late-time afterglow energies (and therefore became available only with the launching Swift). We have shown that there is a large uncertainty in the values of both $\kappa$ and $f$, which translates into a corresponding uncertainty in the value of the $\gamma$-ray efficiency, $\epsilon_{\gamma}$. In the following discussion we make the conservative assumption that we observe most of the emitted energy in $\gamma$-rays $\left(\epsilon_{\mathrm{obs}, \mathrm{GRB}} \approx 1\right)$; if a significant fraction of the total radiated energy is emitted outside of the observed photon energy range (e.g., at higher energies) then this would increase the required value of $\epsilon_{\gamma}$ (see equation [13]).

The kinetic energy of the afterglow shock has been estimated in the pre-Swift era using broad-band afterglow fits for a small number of GRB sources that had the best available broad-band afterglow data, yielding a typical value of $\kappa \sim 1$ with a large scatter of almost

\footnotetext{
${ }^{10}$ It is in principle also conceivable that the baryon-poor outflow could develop an internal structure that would correspond to the two outflow components considered here, but there are at present no quantitative results to support this conjecture.
} 
an order of magnitude (see $\S 2.3$ ). Substituting the values of the microphysical parameters $\left(\epsilon_{e}, \epsilon_{B}\right.$ and $\left.p\right)$ that were derived from these fits into our equations for $E_{\mathrm{k} \text {,iso generally yielded }}$ somewhat lower values of $\kappa$. Finally, using our equations with fiducial values of the microphysical parameters $\left(\epsilon_{e}=0.1, \epsilon_{B}=0.01\right.$, and $\left.p=2.2\right)$ gives a typical value of $\kappa \sim 0.1-0.2$, both for the pre-Swift and the Swift GRB samples that we use, with a moderate scatter. Specifically, for our pre-Swift (Swift) sample, $\left\langle\log _{10} \kappa\right\rangle=-0.75(-0.82)$ corresponding to $\kappa=0.18(0.15)$ and $\sigma_{\log _{10} \kappa}=0.60$ (0.63). Obviously, the choice of fiducial values for the microphysical parameters is somewhat arbitrary and affects the resulting value of $\kappa$. Higher values of the microphysical parameters $\epsilon_{e}$ and $\epsilon_{B}$ (e.g., $\epsilon_{e} \approx 0.3, \epsilon_{B} \approx 0.08$ ) are required in order to obtain an average value of $\log \kappa$, using our equations, similar to that derived from pre-Swift broad-band afterglow fits. Altogether, there is almost an order-of-magnitude uncertainty in the typical value of $\kappa$ (which ranges from $\sim 0.1-0.2$ to $\sim 1$ ).

Even if we adopt the high typical values of $\kappa(\sim 1)$ inferred from pre-Swift afterglow fits, it is important to keep in mind that these values have been estimated on the basis of the standard assumptions of afterglow theory. Changing these assumptions would modify the inferred value of $\kappa$ for the same fits. For example, as pointed out by Eichler \& Waxman (2005), if only a fraction $\xi_{e}<1$ of the electrons are accelerated to relativistic energies, then there is a degeneracy where the same observable quantities are obtained for $\epsilon_{e} \rightarrow \xi_{e} \epsilon_{e}$, $\epsilon_{B} \rightarrow \xi_{e} \epsilon_{B}, n_{\mathrm{ext}} \rightarrow \xi_{e}^{-1} n_{\mathrm{ext}}$, and $E_{\mathrm{k} \text {,iso }} \rightarrow \xi_{e}^{-1} E_{\mathrm{k}, \text { iso }}$. Since this increases the inferred value of $E_{\mathrm{k}, \text { iso }}$ by a factor of $\xi_{e}^{-1}, \kappa$ is reduced by the same factor in comparison with the estimate from the standard theory (which uses $\xi_{e}=1$ ).

An alternative way of reducing the inferred value of $\epsilon_{\gamma}$ was proposed by Peng et al. (2005) in the context of the pre-Swift observations. Specifically, they considered a twocomponet outflow model with parameters that effectively corresponded to the parameter $f$ having a value $<1$. This parameter choice appears to be inconsistent with Swift's detection of an early flattish decay phase in the X-ray light curve, which, when interpreted in the context of the standard afterglow theory as arising from a gradual increase with time of $E_{\mathrm{k}, \text { iso }}$, typically implies $f \sim 10$ (and, in some cases, values of $f$ that are as high as $\sim$ $\left.10^{2}-10^{3}\right)$. It is worth noting in this connection that the existence of a two-component GRB jet model can be plausibly expected on various theoretical grounds and has been suggested independently on the basis of fits to several pre-Swift afterglows (see discussion in Peng et al. 2005). Furthermore, the Swift observations by no means rule out this model, although they can be used to constrain its parameters. We have illustrated this fact through the fit to the X-ray light curve of GRB 050315 that we performed in $\S 4$ within the framework of this model. This fit has yielded plausible ranges for the kinetic energies and opening angles of the two components as well for as the Lorentz factor of the dominant (wide) component. A key conclusion from this fit is that the kinetic energy of the wide component 
is much larger than that of the narrow one $\left(E_{\mathrm{k}, \mathrm{w}} / E_{\mathrm{k}, \mathrm{n}} \sim 10^{2}\right)$. Physically, the narrow and wide components could conceivably correspond to a baryon-poor, black-hole-driven outflow (Levinson \& Eichler 1993, 2003) and a baryon-rich, disk-driven outflow (Vlahakis \& Königl 2003a,b), respectively, although this remains to be demonstrated. We also note that the twocomponent jet parameters derived in $\S 4$ were based on standard assumptions; they could change if the underlying assumptions (involving, for example, the values and time constancy of the microphysical parameters) were altered.

In conjunction with the $\kappa \sim 1$ estimates of the standard broad-band afterglow fits, values of $f \gtrsim 10$ imply $\gamma$-ray radiative efficiencies $\epsilon_{\gamma} \gtrsim 0.9$. Such high efficiencies would be essentially impossible to achieve in any scheme, such as the internal-shocks model, that tapped the kinetic energy of the outflow for radiation. An alternative possibility that has been discussed in the literature is the direct transfer of Poynting flux (which evidently is also a major contributor to the flow acceleration - e.g., Drenkhahn \& Spruit 2002; Vlahakis \& Königl 2003a) into nonthermal radiation (e.g., Usov 1994; Thompson 1994). It is at present unclear how to assess the efficiency of this process. There are two generic possibilities: dissipative fronts and magnetic reconnection sites. The first option corresponds to overtaking collisions of magnetically dominated relativistic streams and is not expected to result in high radiative efficiencies (e.g., Romanova \& Lovelace 1997; Levinson \& van Putten 1997). The second case would require magnetic field orientation reversals and would most naturally arise in a pulsar-type outflow from a rapidly rotating neutron star (e.g., Spruit et al. 2001). In this case it is, however, still unclear whether radiative efficiencies $\gtrsim 0.9$ could be attained even under the most favorable assumptions about the field reconnection rate (Drenkhahn \& Spruit 2002), and it has in fact been suggested that the reconnection rate might be self-limiting (Lyubarsky \& Kirk 2001).

An "intermediate" situation could prevail if $f \sim 10$ but $\kappa \sim 0.1$, reflecting the possibility that $\kappa$ was overestimated by the pre-Swift afterglow fits (perhaps because some of the assumptions of the standard theory do not hold - e.g., $\xi_{e} \sim 0.1$ rather than $\xi_{e}=1$ ). Alternatively, $\kappa$ could be $\sim 1$ but $f=1$, corresponding to the early flattish decay phase reflecting an increase with time of the X-ray afterglow efficiency $\epsilon_{\mathrm{X}}$ (due, e.g., to $p$ being $<2$ or to an increase with time of $\epsilon_{e}$ or $\epsilon_{B}$; see $\S 3$ ) rather than an early increase in $E_{\mathrm{k} \text {,iso. In }}$ either one of these cases the inferred $\gamma$-ray radiative efficiency would be reduced to $\epsilon_{\gamma} \sim 0.5$. Although this value is less extreme than the estimate discussed in the preceding paragraph, it is worth noting that it is still fairly restrictive for the internal-shocks model, in which it could potentially be attained only if all of the following conditions (already summarized in Peng et al. 2005) are satisfied: (1) the ratio between the maximum and minimum initial Lorentz factors of the ejected shells is large enough $(\gtrsim 10) ;(2)$ the distribution of initial Lorentz factors is sufficiently nonuniform; (3) the shells are approximately of equal mass 
and their number is large enough $(\gtrsim 30)$, and (4) the fraction of the dissipated energy that is deposited in electrons and then radiated away is sufficiently high $\left(\epsilon_{e, \mathrm{GRB}} \epsilon_{\mathrm{rad}, \mathrm{GRB}} \gtrsim 0.5\right)$, with a similar constraint applying to the fraction $\epsilon_{\mathrm{obs}, \mathrm{GRB}}$ of the radiated energy that is emitted as the observed $\gamma$-rays (see Beloborodov 2000 and Kobayashi \& Sari 2001). Only if both $f \sim 1$ and $\kappa \sim 0.1$ were satisfied (which could occur, for example, if $\epsilon_{e}$ or $\epsilon_{B}$ initially increased with time and $\xi_{e}$ were $\sim 0.1$ ) would the inferred value of $\epsilon_{\gamma}$ drop to $\sim 0.1$ and be compatible with the values that are expected to arise under less constrained circumstances in the internal-shocks model.

We are grateful to D. Eichler, E. Ramirez-Ruiz, and P. Kumar for many useful discussions. This research was supported in part by the US Department of Energy under contract number DE-AC03-76SF00515 (J. G.), by NASA Astrophysics Theory Program grant NAG512635 (A. K.), as well as by the US-Israel BSF and by the Schwartzmann University Chair (T. P.). J. G. and A. K. thank the Racah Institute of Physics at the Hebrew University in Jerusalem for hospitality during the early phase of this work.

\section{REFERENCES}

Barthelmy, S. D., et al. 2005, ApJ, 635, 133

Beloborodov, A. M. 2000, ApJ, 539, L25

Berger, E., Kulkarni, S. R., \& Frail, D. A. 2003, ApJ, 590, 379

Blandford, R. D., \& McKee, C. F. 1976, Phys. Fluids, 19, 1130

Blandford, R. D., \& Znajek, R. L. 1977, MNRAS, 179, 433

Bloom, J. S., Frail, D. A., \& Kulkarni, S. R. 2003, ApJ, 594, 674

Daigne F., \& Mochkovitch R. 1998, MNRAS, 296, 275

Drenkhahn, G., \& Spruit, H. .C. 2002, A\&A, 391, 1141

Eichler, D., \& Granot, J. 2005, ApJ, submitted (astro-ph/0509857)

Eichler, D., \& Waxman, E. 2005, ApJ, 627, 861

Fan, Y., \& Piran, T. 2006, preprint (astro-ph/0601054)

Frail, D. A., et al. 2001, ApJ, 562, L55 
Freedman, D. L., \& Waxman, E. 2001, ApJ, 547, 922

Granot, J., \& Kumar, P. 2003, ApJ, 591, 1086

Granot, J., \& Kumar, P. 2006, MNRAS, in press (astro-ph/0511049)

Granot, J., \& Sari, R. 2002, ApJ, 568, 820

Guetta, D., Spada, M., \& Waxman, E. 2001, ApJ, 557, 399

Kobayashi, S., Piran, T., \& Sari, R. 1997, ApJ, 490, 92

Kobayashi, S., \& Sari, R. 2001, ApJ, 551, 934

Kobayashi, S., Zhang, B., Mészáros, P., \& Burrows, D. N. 2005, ApJL, submitted (astro$\mathrm{ph} / 0506157)$

Königl, A., \& Granot, J. 2002, ApJ, 574, 134

Kumar, P, \& Panaitescu, A. 2000, ApJ, 541, L51

Kumar, P. 1999, ApJ, 523, L113

Kumar, P. 2000, ApJ, 538, L125

Kumar, P, \& Piran, T. 2000, ApJ, 535, 152

Levinson, A., \& Eichler, D. 1993, ApJ, 418, 386

Levinson, A., \& Eichler, D. 2003, ApJ, 594, L19

Levinson, A., \& van Putten, M. H. P. M. 1997, ApJ, 488, 69

Lithwick, Y., \& Sari, R. 2001, ApJ, 555, 540

Lloyd-Ronning, N. M., \& Zhang, B. 2004, ApJ, 613, 477

Lyubarsky, Y., \& Kirk, J. G. 2001, ApJ, 547, 437

McKinney, J. C. 2005a, ApJ, submitted (astro-ph/0506368)

McKinney, J. C. 2005b, ApJ, submitted (astro-ph/0506369)

Medvedev, M. V., Fiore, M., Fonseca, R. A., Silva, L. O., \& Mori, W. B. 2005, ApJ, 618, L75

Melatos, A., \& Melrose, D. B. 1996, MNRAS, 279, 1168 
Nousek, J. A., et al. 2005, ApJ, submitted (astro-ph/0508332)

Panaitescu, A., \& Kumar, P. 2001, ApJ, 554, 667

Panaitescu, A., \& Kumar, P. 2001, ApJ, 560, L49

Panaitescu, A., \& Kumar, P. 2002, ApJ, 571, 779

Panaitescu, A., Mészáros, P., Gehrels, N., Burrows, D., \& Nousek, J. 2005, MNRAS, in press

Peng, F., Königl, A., \& Granot, J. 2005, ApJ, 626, 966

Piran, T. 1999, Phys. Rep., 314, 575

Piran, T. 2004, Rev. Mod. Phys., 76, 1143

Piran, T., Kumar, P., Panaitescu, A., \& Piro, L. 2001, ApJ, 560, L167

Romanova, M. M., \& Lovelace, R. V. E. 1997, ApJ, 475, 97

Sari, R., \& Esin, A. A. 2001, ApJ, 548, 787

Sari, R., Piran, T., \& Narayan, R. 1998, ApJ, 497, L17

Smolsky, M. V., \& Usov, V. V. 2000, ApJ, 531, 764

Spruit, H. C., Daigne, F., \& Drenkhahn, G. 2001, A\&A, 369, 694

Tagliaferri, G., et al. 2005, Nature, 436, 985

Thompson, C. 1994, MNRAS, 270, 480

Usov, V. V. 1994, MNRAS, 267, 1035

van Putten, M. H. P. M., \& Levinson, A. 2003, ApJ, 584, 937

Vlahakis, N., \& Königl, A. 2003a, ApJ, 596, 1080

Vlahakis, N., \& Königl, A. 2003b, ApJ, 1104

Vlahakis, N., Peng, F., \& Königl, A. 2003, ApJ, 594, L23

Waxman, E. 1995, PRL, 75, 386

Waxman, E. 2004, ApJ, 606, 988

Waxman, E., \& Bahcall, J. N. 1997, PRL, 78, 2292 
Yost, S. A., Harrison, F. A., Sari, R., \& Frail, D. A. 2003, ApJ, 597, 459

Zhang, B., et al. 2005, ApJ, submitted (astro-ph/0508321) 
Table 1. Estimates of $\kappa$ for pre-Swift GRBs with known redshifts

\begin{tabular}{|c|c|c|c|c|c|c|c|c|c|c|c|c|c|}
\hline $\begin{array}{c}\text { GRB } \\
\#\end{array}$ & $z$ & $\begin{array}{c}L_{\mathrm{X}, \mathrm{iso}, 10 \mathrm{hr}}^{\dagger} \\
\left(10^{46} \mathrm{erg} / \mathrm{s}\right)\end{array}$ & $\begin{array}{c}E_{\gamma, \text { iso }}^{\text {obs }} \\
\left(10^{52} \mathrm{erg}\right)\end{array}$ & $\begin{array}{l}E_{\mathrm{k}, \text { iso, } 10 \mathrm{hr}}^{\mathbf{\top}} \\
\left(10^{52} \mathrm{erg}\right)\end{array}$ & $\begin{array}{c}E_{\mathrm{k}, \text { iso, } 10 \mathrm{hr}}^{(\mathrm{PKO}} \\
\left(10^{52} \mathrm{erg}\right)\end{array}$ & $\begin{array}{c}E_{\mathrm{k}, \text { iso, } 10 \mathrm{hr}}^{(\mathrm{PKO}} \\
\left(10^{52} \mathrm{erg}\right)\end{array}$ & $\begin{array}{c}E_{\mathrm{k}, \text { iso, }}^{(\mathrm{Y} 03) \bigcirc} \bigcirc \\
\left(10^{52} \mathrm{erg}\right)\end{array}$ & $\begin{array}{c}E_{\mathrm{k}, \text { iso }, 1 \mathrm{~d}}^{(\mathrm{Y} 03)} \\
\left(10^{52} \mathrm{erg}\right)\end{array}$ & $\kappa$ ฯ & $\begin{array}{c}\kappa^{\star} \\
(\mathrm{PK} 02)\end{array}$ & $\begin{array}{c}\kappa^{\text {* }} \\
(\mathrm{PK} 02)\end{array}$ & $\begin{array}{l}\kappa_{10 \mathrm{hr}}^{\odot} \\
(\mathrm{Y} 03)\end{array}$ & $\begin{array}{l}\kappa_{1 \mathrm{~d}}^{\diamond} \\
(\mathrm{Y} 03)\end{array}$ \\
\hline 970228 & 0.695 & 0.682 & 1.416 & 22.2 & - & - & - & - & 0.064 & -- & - & - & - \\
\hline 970508 & 0.835 & 0.374 & 0.546 & 12.5 & 1.31 & 4.0 & 1.50 & 1.6 & 0.044 & 0.42 * & $0.14^{*}$ & 0.36 & 0.34 \\
\hline 970828 & 0.985 & 1.76 & 21.98 & 54.8 & - & - & - & - & 0.40 & -- & - & - & - \\
\hline 971214 & 3.418 & 8.96 & 21.05 & 258 & - & - & - & - & 0.082 & -- & - & - & - \\
\hline 980613 & 1.096 & 0.536 & 0.536 & 17.7 & - & - & - & - & 0.030 & -- & - & - & - \\
\hline 980703 & 0.966 & 1.02 & 6.012 & 32.6 & - & - & 61.6 & 13 & 0.18 & -- & - & 0.098 & 0.46 \\
\hline 990123 & 1.600 & 12.83 & 143.8 & 364 & 339 & 22 & - & - & 0.40 & 0.42 & 6.4 & - & - \\
\hline 990510 & 1.619 & 8.209 & 17.64 & 238 & $70^{\star}$ & 9.6 & - & - & 0.074 & $0.25^{\star}$ & 1.8 & - & - \\
\hline 990705 & 0.840 & 0.123 & 25.60 & 4.35 & - & - & - & - & 5.89 & -- & - & - & - \\
\hline 991216 & 1.02 & 18.32 & 53.54 & 510 & - $^{*}$ & 9.9 & - & -- & 0.10 & —* & 5.4 & - & - \\
\hline 000210 & 0.846 & 0.183 & 16.93 & 6.35 & - & - & - & - & 2.67 & -- & - & - & - \\
\hline 000926 & 2.037 & 7.169 & 27.97 & 209 & 36.7 & 3.2 & 272 & 15 & 0.13 & 0.76 & 8.7 & 0.10 & 1.86 \\
\hline 010222 & 1.477 & 13.79 & 85.78 & 389 & 19 * & 16 & - & - & 0.22 & $4.5^{\star}$ & 5.4 & - & - \\
\hline 011211 & 2.14 & 0.886 & 6.723 & 28.5 & - & - & - & - & 0.24 & -- & - & - & - \\
\hline 020405 & 0.698 & 1.729 & 7.201 & 53.9 & - & - & - & - & 0.13 & -- & - & - & - \\
\hline 020813 & 1.254 & 12.12 & 77.50 & 344 & - & - & - & - & 0.23 & -- & - & - & - \\
\hline 021004 & 2.323 & 6.536 & 5.560 & 191 & - & - & - & - & 0.029 & -- & - & - & - \\
\hline
\end{tabular}

Note. - Estimates for $E_{\mathrm{k}, \text { iso }}(10 \mathrm{hr})=E_{\mathrm{k}, \text { iso, } 10 \mathrm{hr}}$ and $\kappa=E_{\gamma, \text { iso }}^{\mathrm{obs}} / E_{\mathrm{k}, \text { iso,10hr }}$ for the GRBs with known redshift from the Bloom et al. (2003) and Berger et al. (2003) samples. ${ }^{\dagger} L_{\mathrm{X}, \text { iso }, 10 \mathrm{hr}}=L_{\mathrm{X} \text {,iso }}\left(10 \mathrm{hr}\right.$ ) from Table 2 of Berger et al. (2003), ${ }^{\ddagger} E_{\gamma, \text { iso }}^{\text {obs }}$ in the $20-2000 \mathrm{keV}$ range form Table 2 of Bloom et al. (2003), ${ }^{\boldsymbol{T}}$ calculated using eq. (5) with our fiducial values of the microphysical parameters: $\epsilon_{e}=0.1, \epsilon_{B}=0.01$, and $p=2.2$. substituting the best fit values of the microphysical parameters from

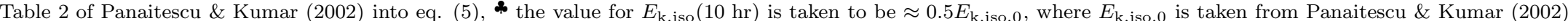
and the factor of $\approx 0.5$ accounts for the average factor by which the energy is reduced relative to $E_{\mathrm{k}, \text { iso }, 0}$ due to radiative losses (A. Panaitescu, personal communication), * these values are for a fit to a uniform external density; PK02 get a significantly better fit to a wind density for which we derive $\kappa=0.47$ and 0.17 instead of 0.42 and 0.14 , respectively, ${ }^{\star}$ in these cases Panaitescu \& Kumar (2002) find $p<2$ which introduces an uncertainty through the extrapolation that is involved in the expression for the numerical coefficient in eq. (9); for GRB 991216 PK02 find that the X-ray emission is from electrons with $\gamma_{e}>\gamma_{\text {max }}$ where $d N / d \gamma_{e} \propto \gamma_{e}^{-q}$ with $p<2<q$ so that we cannot readily substitute their results into our equations, ${ }^{\odot}$ substituting the best fit values of the microphysical parameters from Table 1 of Yost et al. (2003) into eq. (5), $\diamond E_{\mathrm{k}, \text { iso }, 1 \mathrm{~d}}=E_{\mathrm{k}, \text { iso }}(1$ day $)=E_{\gamma, \text { iso }}^{\text {obs }} / \kappa_{1 \mathrm{~d}}$ from Table 1 of Yost et al. $(2003) . \diamond \diamond \kappa_{1 \mathrm{~d}}=E_{\gamma, \text { iso }}^{\text {obs }} / E_{\mathrm{k}, \text { iso }, 1 \mathrm{~d}}$. 
Table 2. Estimates of $f$ and $\kappa$ for Swift GRBs with known redshifts

\begin{tabular}{llccccccc}
\hline \hline GRB \# & $z$ & $\begin{array}{c}L_{\mathrm{X}, \text { iso,10hr }}^{\dagger} \\
\left(10^{46} \mathrm{erg} / \mathrm{s}\right)\end{array}$ & $\begin{array}{c}E_{\gamma, \text { iso }}^{\text {obs }} \\
\left(10^{52} \mathrm{erg}\right)\end{array}$ & $\begin{array}{c}E_{\mathrm{k}, \text { iso, } 10 \mathrm{hr}}^{\mathbf{q}} \\
\left(10^{52} \mathrm{erg}\right)\end{array}$ & $p^{\boldsymbol{4}}$ & $\kappa^{\boldsymbol{*}}$ & $f_{\min }^{\odot}$ & $f_{\max }^{\odot}$ \\
\hline 050126 & 1.29 & 0.12 & 2.2 & 6.86 & 3 & 0.055 & - & - \\
050315 & 1.949 & 16 & 18 & 699 & 2.1 & 0.034 & 11 & 29 \\
050318 & 1.44 & 0.60 & 3.9 & $\gtrsim 28.4$ & 2.1 & $\lesssim 0.18$ & 4.2 & 170 \\
050319 & 3.24 & 5.1 & 12.1 & $\gtrsim 118$ & 2.6 & $\lesssim 0.039$ & 12 & 76 \\
050401 & 2.90 & 9.8 & 137 & 433 & 2.1 & 0.41 & 5.6 & 14 \\
050408 & 1.236 & 1.4 & 2.9 & 38.5 & 2.3 & 0.058 & - & - \\
$050416 \mathrm{~A}$ & 0.6535 & 0.091 & 0.09 & 4.51 & 2.1 & 0.026 & 2.2 & 9.9 \\
050505 & 4.3 & 2.3 & 89 & $\gtrsim 59.0$ & 2.6 & $\lesssim 0.58$ & 19 & 1800 \\
$050525 \mathrm{~A}$ & 0.606 & 0.12 & 3.1 & $\gtrsim 3.96$ & 2.4 & $\lesssim 0.47$ & 2.1 & 5.9 \\
050603 & 2.821 & 1.1 & 126 & $\gtrsim 29.6$ & 2.4 & $\lesssim 2.57$ & - & - \\
\hline
\end{tabular}

Note. - The estimates for $f=E_{\mathrm{k}, \text { iso }, 10 \mathrm{hr}} / E_{\mathrm{k}, \text { iso }, 0}^{\mathrm{obs}}$ and $\kappa=E_{\gamma, \text { iso }}^{\mathrm{obs}} / E_{\mathrm{k}, \text { iso }, 10 \mathrm{hr}}$ for the GRBs with known redshift from the Nousek et al. (2005) sample; ${ }^{\dagger} L_{\mathrm{X}, \text { iso, } 10 \mathrm{hr}}=L_{\mathrm{X} \text {,iso }}(10 \mathrm{hr})$ in the $2-10 \mathrm{keV}$ range at (both the time and the photon energies measured in the cosmological frame of the GRB) from Table 2 of Nousek et al. (2005), ${ }^{\ddagger} E_{\gamma, \text { iso }}^{\text {obs }}$ in the $20-2000 \mathrm{keV}$ range (in the GRB's cosmological frame) form Table 2 of Nousek et al. (2005), ` calculated using eq. (5) with $\epsilon_{e}=0.1, \epsilon_{B}=0.01$, and the values of $p$ from this Table, " estimated using the measured spectral slope in the X-rays (Nousek et al. 2005) and attempting to fit it into the range $2<p<3$ if allowed within the errors on the spectral slope, $\boldsymbol{\star}^{\boldsymbol{\alpha}} \kappa=E_{\gamma, \text { iso }}^{\mathrm{obs}} / E_{\mathrm{k} \text {,iso, } 10 \mathrm{hr}}$ estimated using the values from this Table, ${ }^{\varnothing} f_{\min }$ and $f_{\max }$ are taken from Table 3 of Nousek et al. (2005), and are estimated using the measured X-ray flux at $t_{\text {break,1 }}$, and the extrapolated X-ray flux at $T_{\mathrm{GRB}}$, respectively (see Nousek et al. 2005 for details). 


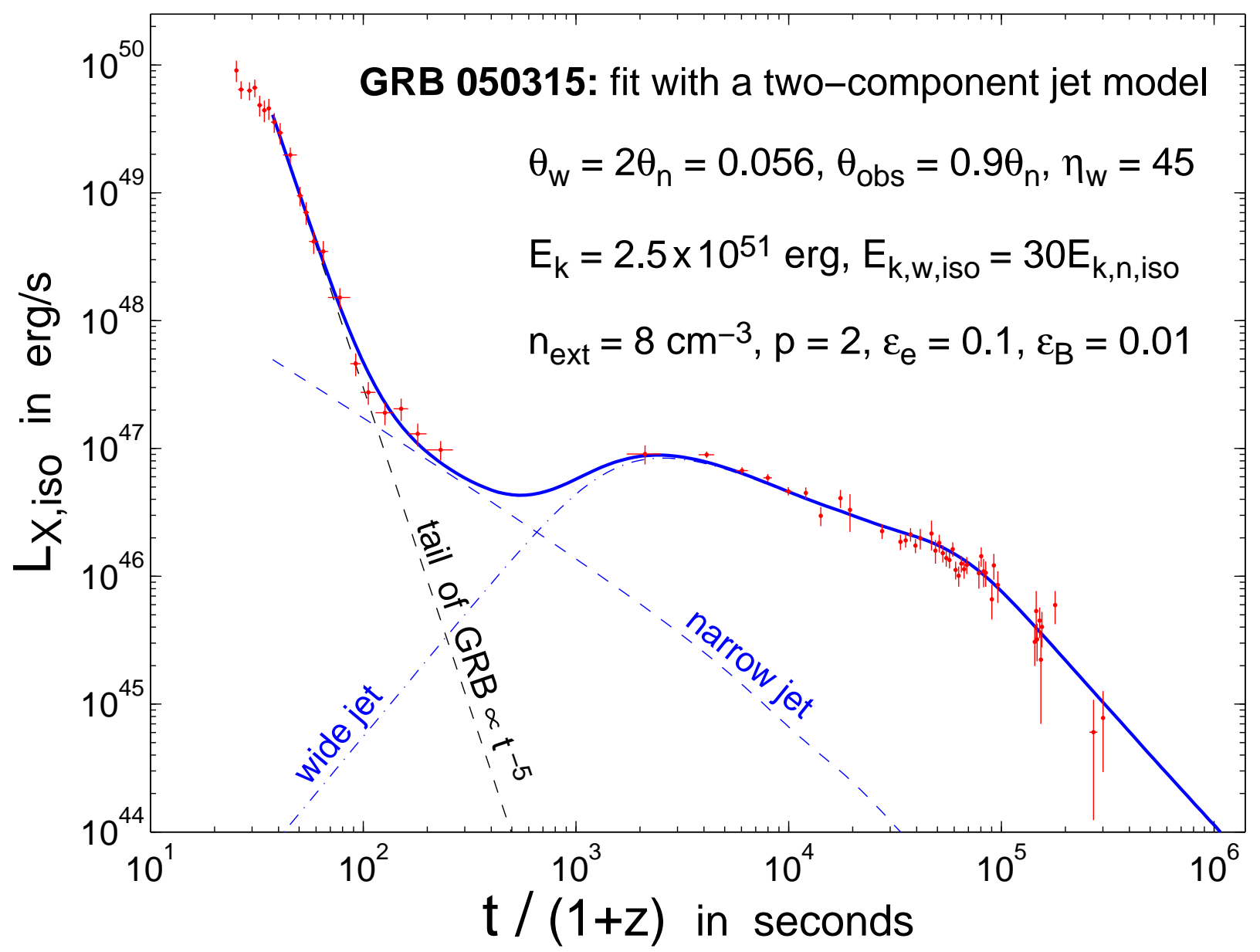

Fig. 1. - Tentative fit to the X-ray light curve of GRB 050315 (from Nousek et al. 2005) with the two-component jet model. The numerical code used to calculate the light curve is essentially Model 1 of Granot \& Kumar (2003), which neglects the lateral spreading of the jet and includes synchrotron self-Compton (SSC) emission. In addition to the total light curve (thick solid line) we also show the separate contributions of the different components: the tail of the prompt emission $\left(\propto t^{-5}\right)$, the narrow outflow, and the wide outflow. Here $E_{\mathrm{k}}=E_{\mathrm{k}, \mathrm{w}}+E_{\mathrm{k}, \mathrm{n}}$ is the total kinetic energy of the two components. The narrow and wide components occupy the non-overlapping ranges $\theta<\theta_{\mathrm{n}}$ and $\theta_{\mathrm{n}}<\theta<\theta_{\mathrm{w}}$, respectively, in the polar angle $\theta$ (measured from the symmetry axis); $\theta_{\mathrm{obs}}$ is the viewing angle with respect to this axis. 


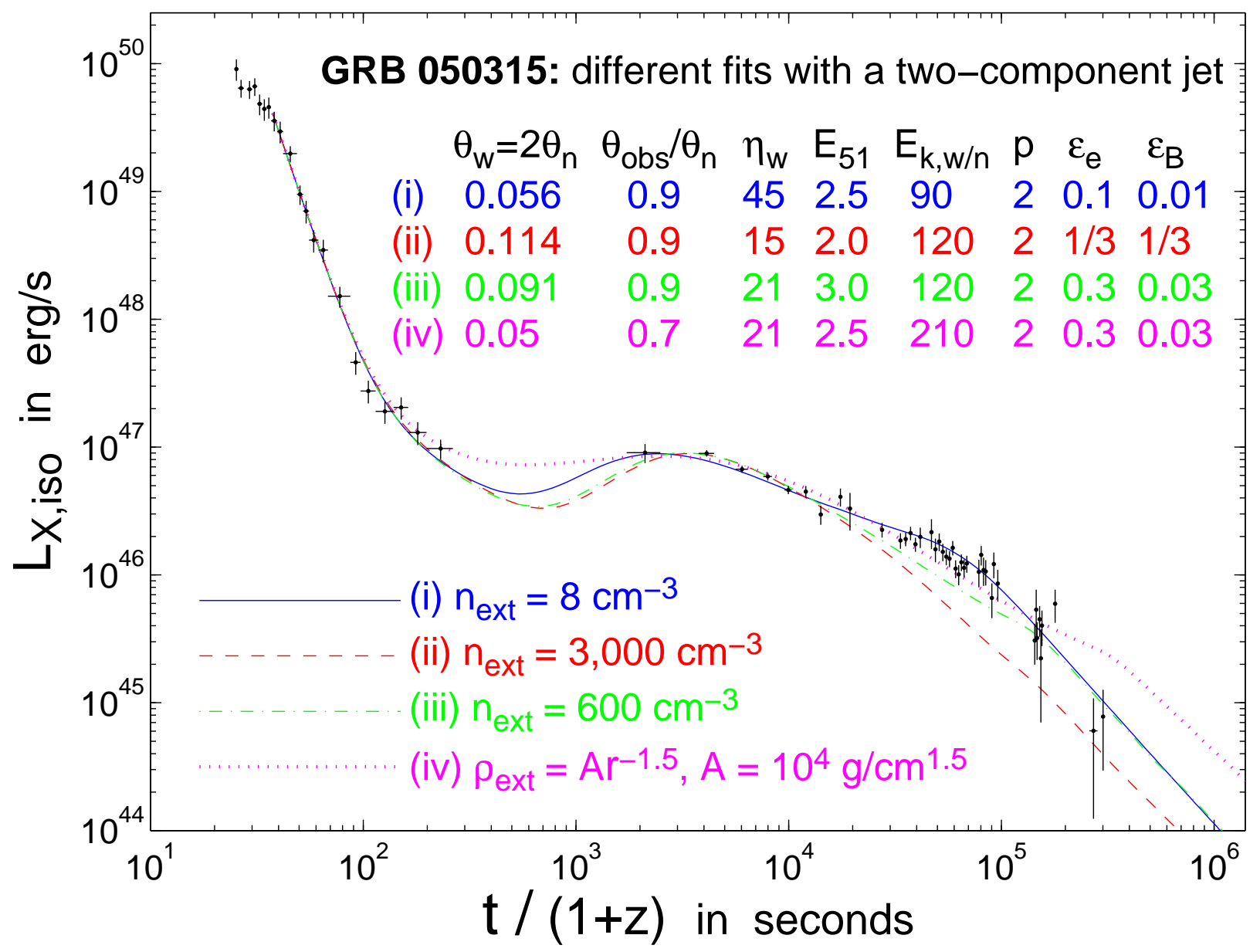

Fig. 2.- The effects of varying the two-component jet model parameters with respect to those of the fit shown in Fig. 1 (reproduced in this figure by the solid line). Here $E_{51}=$ $E_{\mathrm{k}} /\left(10^{51} \mathrm{erg}\right)\left(\right.$ with $\left.E_{\mathrm{k}}=E_{\mathrm{k}, \mathrm{w}}+E_{\mathrm{k}, \mathrm{n}}\right)$, whereas $E_{\mathrm{k}, \mathrm{w} / \mathrm{n}}$ denotes the ratio $E_{\mathrm{k}, \mathrm{w}} / E_{\mathrm{k}, \mathrm{n}}$ of the kinetic energies of the wide and narrow components. 